\title{
Fast Stokes flow simulations for geophysical-geodynamic inverse problems and sensitivity analyses based on reduced order modeling
}

\author{
O. Ortega-Gelabert ${ }^{1}$, S. Zlotnik ${ }^{1}$, J.C. Afonso ${ }^{2,3}$, and P. Díez ${ }^{1}$ \\ ${ }^{1}$ Laboratori de Càlcul Numèric (LaCàN), Departament d'Enginyeria Civil i Ambiental, Universitat \\ Politècnica de Catalunya, Barcelona, Spain. \\ ${ }^{2}$ Department of Earth and Planetary Sciences, Macquarie University, Sydney, Australia. \\ ${ }^{3}$ Centre for Earth Evolution and Dynamics, Department of Geosciences, University of Oslo, Norway.
}

\section{Key Points:}

- An efficient method for producing ultra-fast solutions of the 3D Stokes problem for mantle convection is presented.

- This novel method is particularly well-suited for joint geodynamic-geophysical probabilistic inverse problems and studies of dynamic topography.

- The general character of the method makes it suitable to tackle a large range of probabilistic inverse problems where fast solutions for a complex forward problem are needed.

Corresponding author: Sergio Zlotnik, sergio.zlotnik@upc.edu 


\begin{abstract}
Markov Chain Monte Carlo (MCMC) methods have become standard in Bayesian inference and multi-observable inversions in almost every discipline of the Earth sciences. In the case of geodynamic and/or coupled geophysical-geodynamic inverse problems, however, the computational cost associated with the solution of large-scale 3D Stokes forward problems has rendered probabilistic formulations impractical.
\end{abstract}

Here we present a novel and extremely efficient method to produce ultra-fast solutions of the 3D Stokes problem for MCMC simulations. Our approach combines the individual benefits of Reduced Basis techniques, goal-oriented error formulations and MCMC algorithms to produce an accurate and computationally efficient surrogate for the forward problem. Importantly, the surrogate adapts itself during the MCMC simulation according to the history of the chain and the goals of the inversion. This maximizes the efficiency of the forward problem and removes the need for pre-inversion offline computations to build a surrogate. We demonstrate the benefits and limitations of the method with several numerical examples and show that in all cases the computational cost is of the order of $<1 \%$ compared to a traditional MCMC approach. The method is general enough to be applied to a range of problems, including uncertainty quantification/propagation, adjoint-based geodynamic inversions, sensitivity analyses in mantle convection problems, as well as in the creating surrogate models for complex forward problems (e.g. heat transfer, seismic tomography, Magnetotellurics).

\title{
1 Introduction
}

The last 20 years have witnessed an outstanding increase in computing power, datafusion techniques, and geophysical data acquisition programs worldwide; a trend that is likely to accelerate in the next few decades. These advances now allow the Solid Earth community to pursue massive data-driven simulations and probabilistic joint inversions for the physical state and geodynamic evolution of the Earth's interior with unprecedented complexity and resolution. In fact, the current state of affairs is such that the boundaries between the fields of geodynamics and inverse geophysical theory, traditionally viewed as separate disciplines, are becoming increasingly blurred and symbiotic (e.g., Boschetti \& Moresi, 2001; Bunge et al., 2003; Simmons et al., 2009, 2010; Afonso, Rawlinson, et al., 2016; Baumann et al., 2014; van Dinther et al., 2013; Baumann \& Kaus, 2015; Li et al., 2017; Colli et al., 2018). The fact that these disciplines have evolved in isolation from each other is easily understood when we consider the fundamentally different timescales $\tau$ involved in the propagation and measurement of the signals relevant to each discipline (e.g. $10^{0} \leq \tau \lesssim 10^{3} \mathrm{sec}$ for seismic waves and gravity anomalies; $10^{10} \leq \tau \lesssim 10^{16}$ sec for postglacial rebound and mantle convection). Consequently, geophysical inversions have focused on the "static" or present-day structure of the planet (as seen today), ignoring time-dependent "dynamic" contributions associated e.g. with motion in the mantle. Geodynamic studies, on the other hand, are typically aimed at unravelling slow time-dependent (or evolutionary) processes such as subduction dynamics, mantle convection and the long-term evolution of surface topography.

For many purposes, the separation of these problems is an appropriate and practical working scheme. For instance, there is no need for considering strain rates or density distributions inside the mantle when inverting teleseismic traveltimes for seismic velocity anomalies beneath an array. However, it is well known that models constrained by single datasets typically fail at providing satisfactory fits to other observables (Forte, 2007; Afonso, Fullea, Yang, et al., 2013; Afonso, Rawlinson, et al., 2016). Clearly, Earth models that are simultaneously constrained by multiple and complementary observables are necessary to bring a step-change in our understanding of e.g. the nature (i.e. temperature, composition, architecture) and evolution of the 
lithosphere, its interaction with the sub-lithospheric mantle, and the forces driving tectonism and plate motion. Aligned with this, the current trend of 1) working with multiple datasets in joint inversions, 2) increasing the complexity (i.e. realism) of Earth models, and 3) making these models more comprehensive, explicative and predictive, forces us to consider a variety of physiochemical processes and their couplings at a range of temporal and spatial scales. Joint inversions of long-wavelength gravity and seismic data at global scale represents a classic example pioneered by Forte et al. (1994) and more recently expanded in Simmons et al. (2010). Despite being one of the most advanced approaches to date, these global studies were necessarily based on linearized deterministic inversions, and therefore not well suited for quantifying the associated uncertainties, non-linearities, and/or rigorously considering the stochastic nature of the data noise.

Probabilistic formulations of the inverse problem (Tarantola, 2005; Mosegaard \& Tarantola, 2002; Gregory, 2005; Kaipio \& Somersalo, 2007) represent an attractive option that can overcome some or all of these difficulties. However, they typically rely on sampling techniques that require solving the forward problem many times, thus posing a real challenge when the forward problem is computationally expensive (as in the case of 3D Stokes flow with varying viscosity). Recently, Baumann et al. (2014) and Baumann and Kaus (2015) demonstrated the viability of a probabilistic formulation for the geodynamic inverse problem incorporating realistic assumptions about the mechanical behaviour of the lithosphere and upper mantle. These authors used this approach to constrain rheological parameters and subsurface density at lithospheric scales. As in most probabilistic formulations of complex inverse problems, these authors relied on sampling techniques to characterize the posterior distribution (solution to the inverse problem) of the unknown parameters. Since the forward problem is computationally expensive and needs to be solved many times during a simulation, the numerical domain had to be kept relatively small and access to massive computing clusters was necessary. Moreover, their present implementation relies heavily on a priori knowledge of the temperature and compositional structure of the model; something that is hard to come by in most regions of the world. As Baumann and Kaus (2015) pointed out, integrated approaches that jointly invert or model a number of datsets sensitive to the thermochemical structure of the Earth (e.g., Khan et al., 2008, 2011; Afonso, Fullea, Griffin, et al., 2013; Afonso, Fullea, Yang, et al., 2013; Afonso, Moorkamp, \& Fullea, 2016) would constitute a more appropriate and generally applicable approach. In this context, the recent work of Afonso, Fullea, Griffin, et al. (2013),Afonso, Fullea, Yang, et al. (2013), and Afonso, Rawlinson, et al. (2016) has made significant progress towards such goal by presenting a multi-observable probabilistic inversion method that simultaneously invert the most appropriate datasets (with the necessary complementary sensitivities) for the temperature and compositional structure of the lithosphere and upper mantle: Rayleigh wave dispersion data, teleseismic P and $\mathrm{S}$ traveltimes, gravity anomalies, geoid height, satellite-derived gravity gradients, surface heat flow, and absolute elevation; $\mathrm{P}$-wave receiver functions have also been implemented recently (Tork Qashqai et al., 2016, 2018). Although Afonso, Rawlinson, et al. (2016) included dynamic contributions to absolute elevation and gravity observables from the instantaneous sublithospheric flow, their implementation of the Stokes forward problem was inefficient and based on a number of simplifying assumptions to make the problem tractable in the probabilistic framework. In order to exploit the full capabilities of joint geophysical-geodynamic probabilistic inversions, a more efficient implementation of the Stokes flow problem for large-scale models is required.

The main goal of this work is to formulate an efficient methodology within the framework of the Reduced Basis (RB) method (Quarteroni et al., 2016; Chinesta \& Ladevèze, 2014; Hesthaven et al., 2016). The main idea is to employ a RB approach to construct an accurate approximation or "surrogate" of the true forward problem (i.e. 3D Stokes flow problem). This surrogate is then used to generate samples of 
the posterior distribution (i.e. the solution to the inverse problem) at a much lower computational cost. To guarantee an accurate surrogate, a formal estimation of the error introduced by the RB approximation is defined and used to decide whether the $\mathrm{RB}$ needs to be enriched/updated with additional high-fidelity forward problems. In contrast to traditional RB implementations, where the problem is split into offline (creation of the Basis) and online (compute the low-cost solution) stages, here the RB is created online, guided by the inversion itself. Taking advantage of the ergodicity and stationarity properties of Markov chains, we create, use and enrich/update the surrogate during the actual inversion (i.e. the sampling of the posterior distribution). We refer to Peherstorfer et al. (2018) for a recent review on the use of surrogates in probabilistic inversion and optimization problems. We emphasize here that although our focus is on the application of RB to the solution of Stokes flow in the context of multi-observable probabilistic inversions, our strategy is also relevant to problems dealing with uncertainty quantification/propagation, adjoint-based geodynamic inversions and sensitivity analyses in mantle convection problems. Perhaps more importantly, the same RB strategy can be used to create and use surrogate models for other complex forward problems of interest (e.g. heat transfer, seismic tomography, Magnetotellurics).

In the following, we first review the formulation of the probabilistic inverse problem in Section 2. Next, the forward problem and the general RB approach to produce a fast and accurate surrogate approximation are described in Section 3. Several numerical examples to illustrate the benefits and limitations of the method are presented in Sections 4 and 5. Finally, we conclude the paper with a brief discussion of some practical aspects of our approach relevant to real inversions in Sections 6 and 7.

\section{Inverse problem}

\subsection{General background}

Inverse problem theory provides the formalism to obtain information on unobservable properties of a system from observational data and prior information. The probabilistic approach to an inverse problem is based on the idea of recasting the inverse problem in the form of a statistical inference problem (cf. Kaipio \& Somersalo, 2005; Tarantola, 2005; Mosegaard \& Tarantola, 2002; Gregory, 2005). In doing so, all available information (both observational and prior) is combined with physical theories describing the relationships between model parameters and observations to obtain complete probability distributions over the (unobservable) parameters of interest rather than single "best-fitting" models. These probability distributions represent the most general solution to the inverse problem (i.e. they embody all we know about the system) and can be used to make statistical inferences about the system's constitutive parameters and their associated uncertainties.

In this context, Bayesian statistical inference is widely used these days to solve probabilistic inverse problems with the help of Markov Chain Monte Carlo (MCMC) methods (Tarantola, 2005; Mosegaard \& Tarantola, 2002; Gregory, 2005; Gilks et al., 1996; Afonso, Fullea, Griffin, et al., 2013; Afonso, Fullea, Yang, et al., 2013; Afonso, Rawlinson, et al., 2016). In this approach, the solution to a parameter inference problem (i.e. inversion for model's parameters) is represented by a Probability Density Function (PDF) in the model's parameter space known as the posterior PDF, which can be represented as

$$
\sigma(\mathbf{m}) \propto \rho(\mathbf{m}) L(\mathbf{m})
$$

where $\rho(\mathbf{m})$ is the prior PDF describing our prior knowledge about the model parameters independent of the observed data and $L(\mathbf{m})$ is the so-called likelihood function, 
which measures how well a specific set of model parameters $\mathbf{m}$ fits the observed data d (Tarantola, 2005; Mosegaard \& Tarantola, 2002).

One of the advantages of probabilistic formulations is that they represent a natural platform to include uncertainties in both input data and modelling approach. In the simple case where Gaussian errors contaminate both predicted and observed data, $L(\mathbf{m})$ can be expressed as (e.g., Tarantola, 2005)

$$
L(\mathbf{m}) \propto \exp (-e(\mathbf{m}))
$$

where $e(\mathbf{m})$ is the sum-of-squares function, which can be interpreted as a misfit function between observed and predicted data,

$$
e(\mathbf{m})=\frac{1}{2}\left(\mathbf{g}(\mathbf{m})-\mathbf{d}^{\mathrm{obs}}\right)^{\top} \mathrm{C}_{\mathrm{D}}^{-1}\left(\mathbf{g}(\mathbf{m})-\mathbf{d}^{\mathrm{obs}}\right)
$$

where $C_{D}=C_{d}+C_{T}$ (i.e. the total covariance matrix is the sum of the covariance matrices describing observation $\mathrm{C}_{\mathrm{d}}$ and modelling $\mathrm{C}_{\mathrm{T}}$ uncertainties) and $\mathbf{g}(\mathbf{m})$ is generally a nonlinear operator that solves the mapping from model to data space (also known as the forward problem). In the case of independent uncertainties, this leads to

$$
e(\mathbf{m})=\frac{1}{2} \sum_{i=1}^{n_{d}}\left(\frac{g_{i}(\mathbf{m})-d_{i}{ }^{\mathrm{obs}}}{\sigma_{\mathrm{D}_{i}}}\right)^{2}
$$

where $\sigma_{\mathrm{D}_{i}}$ for $i=1, \ldots, n_{d}$ are the diagonal entries of $\mathrm{C}_{\mathrm{D}}$.

The prior PDF encodes everything we know about the parameter space (i.e. the probabilities of $\mathbf{m}$ ) prior to considering $\mathbf{d}^{\text {obs }}$ and it represents an important part of any Bayesian inference problem. If the prior information is vague then the prior distribution is a very broad/flat distribution. Conversely, narrow distributions indicate that prior knowledge on the respective parameters is accurate. Gaussian, Laplacian, Poisson, uniform and Jeffreys' priors are the most common options in the literature (cf. Mosegaard \& Tarantola, 2002; Kaipio \& Somersalo, 2005). Assuming that priors for all parameters are Gaussian distributions, $m_{i} \sim \mathrm{N}\left(\nu_{i}, \sigma_{m_{i}}^{2}\right)$, it follows that

$$
\rho(\mathbf{m}) \propto \exp (-s(\mathbf{m}))
$$

with

$$
s(\mathbf{m})=\frac{1}{2}(\mathbf{m}-\boldsymbol{\nu})^{\top} \mathrm{C}_{\mathbf{M}}^{-1}(\mathbf{m}-\boldsymbol{\nu})
$$

where $\boldsymbol{\nu}$ is the vector of mean values for the parameters and $\mathrm{C}_{\mathbf{M}}$ the covariance matrix describing prior uncertainties. For uncorrelated prior uncertainties, Eq. (6) reads

$$
s(\mathbf{m})=\frac{1}{2} \sum_{i=1}^{n_{m}}\left(\frac{m_{i}-\nu_{i}}{\sigma_{m_{i}}}\right)^{2}
$$

where $n_{m}$ is the number of parameters and $\nu_{i}$ and $\sigma_{m_{i}}$ the prior's mean and standard deviation for parameter $m_{i}$, respectively.

We can now insert equations (2) and (5) into equation (1) to obtain an expression for the posterior PDF,

$$
\sigma(\mathbf{m}) \propto \exp \left(-\frac{1}{2}\left[\sum_{i=1}^{n_{m}}\left(\frac{m_{i}-\nu_{i}}{\sigma_{m_{i}}}\right)^{2}+\sum_{i=1}^{n_{d}}\left(\frac{g_{i}(\mathbf{m})-d_{i}{ }^{\mathrm{obs}}}{\sigma_{\mathrm{D}_{i}}}\right)^{2}\right]\right)
$$


assuming independence of prior and observations.

For linear (or linearizable) forward problems and Gaussian uncertainties in both model and data spaces, equation (8) is itself a multivariate Gaussian and very efficient optimization algorithms can be used to obtain its mathematical expectations or representative statistics (e.g. maximum likelihood). Here we are interested in largedimensional nonlinear problems, which can result in complex posteriors with no single minimum/maximum. In these cases, stochastic sampling techniques based on MCMC algorithms are general and powerful options to obtain accurate representations of the full posterior (cf. Tarantola, 2005; Mosegaard \& Tarantola, 2002; Gregory, 2005; Gilks et al., 1996; Afonso, Fullea, Griffin, et al., 2013; Afonso, Fullea, Yang, et al., 2013; Afonso, Rawlinson, et al., 2016).

\subsection{MCMC and the Metropolis algorithm}

In this work we estimate the posterior PDF $\sigma(\mathbf{m})$ of a set of model parameters $\mathbf{m}$ using a MCMC approach based on the Metropolis-Hastings algorithm (Metropolis et al., 1953; Hastings, 1970). The main idea is to generate a (guided) random walk in the parameter space $\left\{\mathbf{m}_{1}, \mathbf{m}_{2}, \ldots, \mathbf{m}_{n}\right\}$ such that, when long enough, histograms of the relative frequencies of the parameters approximate $\sigma(\mathbf{m})$. To do this, random samples of $\mathbf{m}$ are generated either directly from its prior distribution $\rho(\mathbf{m})$ or using a proposal distribution that is easy to sample. In this case, the random walk would simply approximate the prior $\rho(\mathbf{m})$. To sample $\sigma(\mathbf{m})$ instead, each proposed model is combined with its respective likelihood $L(\mathbf{m})$ and subject to an acceptance test based on the value of the so-called Metropolis ratio $\alpha$,

$$
\alpha\left(\mathbf{m}_{\text {old }}, \mathbf{m}_{\text {new }}\right)=\frac{\sigma\left(\mathbf{m}_{\text {new }}\right)}{\sigma\left(\mathbf{m}_{\text {old }}\right)}
$$

where $\mathbf{m}_{\text {old }}$ and $\mathbf{m}_{\text {new }}$ refer to the current state in chain and the proposed state, respectively. The Metropolis ratio compares their corresponding posteriors: if $\alpha \geqslant 1$, the proposed model is accepted; otherwise, it is accepted with probability $\alpha$. If a proposed model is not accepted, the random walk remains at the current value and a new proposed model is generated. When this process is repeated many times (i.e. the number of samples is large enough), the Metropolis algorithm guarantees that the relative occurrence of a parameter $m$ in the random walk is proportional to its posterior density. In other words, a histogram of relative occurrence of a parameter $m$ represents the marginal probability distribution of that parameter.

Despite its many advantages relative to traditional matrix-based inversions (e.g., Tarantola, 2005; Afonso, Fullea, Griffin, et al., 2013; Afonso, Moorkamp, \& Fullea, 2016), the use of MCMC methods to solve inverse problems has the drawback of having to evaluate $L(\mathbf{m})$ at each inversion step, which involves computing a full forward problem, thousands to millions of times. Consequently, the possibility of using a probabilistic approach to solve an inverse problem relies entirely on having efficient and accurate solvers for the forward problems (Section 3.2).

\section{Forward problem}

\subsection{Problem statement}

A first-order description of the dynamic behaviour of the mantle and its effect on elevation starts with the formal statement of a Stokes problem (cf. Schubert et al., 
2004),

$$
\left\{\begin{aligned}
-\boldsymbol{\nabla} \cdot\left(\mu \boldsymbol{\nabla}^{\mathbf{S}} \boldsymbol{u}\right)+\boldsymbol{\nabla} p & =\rho \boldsymbol{g} & & \text { in } \Omega \\
\boldsymbol{\nabla} \cdot \boldsymbol{u} & =0 & & \text { in } \Omega \\
\boldsymbol{u} & =\boldsymbol{u}_{D} & & \text { on } \Gamma_{D} \\
-p \boldsymbol{n}+\mu \boldsymbol{n} \cdot \nabla^{\mathbf{S}} \boldsymbol{u} & =\boldsymbol{t} & & \text { on } \partial \Omega \backslash \Gamma_{D}
\end{aligned}\right.
$$

where $\boldsymbol{u}$ and $p$ are velocity and pressure, respectively, $\Omega \subset \mathbb{R}^{3}$ is the computational domain and $\partial \Omega$ is its boundary. The latter is partitioned into Dirichlet, $\Gamma_{D}$, and Neumann boundary types. Body forces are given by $\rho \boldsymbol{g}$, being $\rho$ the density and $\boldsymbol{g}$ the gravity vector. Dirichlet and Neumann boundary conditions are set by $\boldsymbol{u}_{D}$ and $\boldsymbol{t}$. Dynamic viscosity $\mu$ is also considered part of input data. The symmetrized gradient is defined as $\boldsymbol{\nabla}^{\mathbf{S}}=\frac{1}{2}\left(\boldsymbol{\nabla}+\boldsymbol{\nabla}^{\boldsymbol{\top}}\right)$. In what follows, as in (10), we indicate vectors with lowercase bold letters.

The weak form for problem (10) reads: find $(\boldsymbol{u}, p)$ in the suitable spaces $\mathcal{V}$ and $\mathcal{Q}$, such that,

$$
\left\{\begin{aligned}
a(\boldsymbol{w}, \boldsymbol{u})+b(\boldsymbol{w}, p) & =l(\boldsymbol{w}) & & \forall \boldsymbol{w} \in \mathcal{V}, \\
b(\boldsymbol{u}, q) & =0 & & \forall q \in \mathcal{Q},
\end{aligned}\right.
$$

with the operators defined as,

$$
\begin{aligned}
a(\boldsymbol{w}, \boldsymbol{u}) & =\int_{\Omega} 2 \mu \nabla^{\mathbf{s}} \boldsymbol{w}: \nabla^{\mathbf{s}} \boldsymbol{u} d \Omega \\
b(\boldsymbol{u}, q) & =-\int_{\Omega} q \boldsymbol{\nabla} \cdot \boldsymbol{u} d \Omega \text { and } \\
\ell(\boldsymbol{w}) & =\int_{\Omega} \boldsymbol{s} \cdot \boldsymbol{w} d \Omega+\int_{\partial \Omega \backslash \Gamma_{D}} \boldsymbol{v} \cdot \boldsymbol{t} d \Gamma .
\end{aligned}
$$

The solution $\boldsymbol{u}$ is then replaced by a discrete version constructed using a large number of local Finite Element (FE) basis functions (aka shape functions) $N_{i}(\boldsymbol{x})$, for $i=1, \ldots, n_{u}$, that generates the discrete sub-space $\mathcal{V}^{h} \in \mathcal{V}$,

$$
\mathcal{V}^{h}=\operatorname{span}\left\{N_{1}, N_{2}, \ldots, N_{n_{u}}\right\} \text {. }
$$

The vector field $\boldsymbol{u}(\boldsymbol{x})$ is approximated by $\boldsymbol{u}^{h}(\boldsymbol{x}) \in\left[\mathcal{V}^{h}\right]^{3}$ and represented by the vector of nodal values $\mathbf{u} \in \mathbb{R}^{3 n_{u}}$

$$
\boldsymbol{u}(\boldsymbol{x}) \approx \boldsymbol{u}^{h}(\boldsymbol{x})=\sum_{i=1}^{n_{u}} N_{i}(\boldsymbol{x})\left[\begin{array}{l}
{[\mathbf{u}]_{3(i-1)+1}} \\
{[\mathbf{u}]_{3(i-1)+2}} \\
{[\mathbf{u}]_{3(i-1)+3}}
\end{array}\right]
$$

where $[\mathbf{u}]_{j}$, for $j=1, \ldots, 3 n_{u}$, are the nodal velocities.

The scalar field $p(\boldsymbol{x})$ is approximated by $p^{h}(\boldsymbol{x}) \in \mathcal{Q}^{h}$ and represented by the vector of nodal values $\mathbf{p} \in \mathbb{R}^{n_{p}}$

$$
p(\boldsymbol{x}) \approx p^{h}(\boldsymbol{x})=\sum_{i=1}^{n_{p}} \tilde{N}_{i}(\boldsymbol{x})[\mathbf{p}]_{i}
$$

where $[\mathbf{p}]_{i}$ are the nodal pressures and $\tilde{N}_{i}(\boldsymbol{x})$ the corresponding FE basis functions.

Once the discrete subspaces are chosen, system (11) is converted into a linear system of algebraic equations. The matrix associated with this system in the Stokes model is symmetric, with $2 \times 2$ blocks and a null submatrix on the diagonal,

$$
\left[\begin{array}{cc}
\mathbf{K} & \mathbf{G} \\
\mathbf{G}^{\top} & \mathbf{0}
\end{array}\right]\left[\begin{array}{l}
\mathbf{u} \\
\mathbf{p}
\end{array}\right]=\left[\begin{array}{l}
\mathbf{f} \\
\mathbf{0}
\end{array}\right]
$$


The so-called mixed FE problem (15), with velocity and pressure as separate unknowns, is computationally expensive in large 3-dimensional domains, $n_{\mathrm{FE}}=3 n_{u}+$ $n_{p}$ unknowns. Several numerical schemes and preconditioners have been proposed to solve it efficiently. However, the current efficiency of such solvers is still insufficient for large-scale probabilistic inversions. The Reduced Basis technique described next attempts to overcome this problem and is independent of the numerical technique chosen to solve the forward problem.

\subsection{Reduced Basis}

Reduced order techniques, aka Model Order Reduction (MOR), are a family of approximation methodologies based on the common idea of expressing the solution of a problem in terms of a basis of reduced size (Chinesta \& Ladevèze, 2014; Quarteroni et al., 2016; Hesthaven et al., 2016; Ito \& Ravindran, 1998; Ravindran, 2000). "Reduced" in this context refers to a basis (i.e. a family of basis functions) with a cardinal smaller than that resulting from a traditional FE discretization. Of all the available MOR techniques, the so-called Reduced Basis (RB) method (cf. Florentin \& Díez, 2012; Quarteroni et al., 2016; Rozza et al., 2007, 2013), is particularly attractive for our purposes as it is easy to implement and well-suited for situations where physical problems with similar characteristics need to be solved many times for different input parameters. This is the case, for example, in the context of probabilistic inverse problems, where a large number of evaluations of the forward problem (typically solutions to PDEs) are required to approximate the full posterior PDF (Tarantola, 2005; Gregory, 2005; Gilks et al., 1996; Afonso, Fullea, Griffin, et al., 2013; Afonso, Fullea, Yang, et al., 2013). While at the beginning of the MCMC simulation the parameter space may be large, a well-formulated MCMC algorithm will converge quickly to a reduced region of the parameter space where all the forward problems become "similar" (similar in the sense of having similar input parameters and predictions). At this stage, it is wasteful to solve forward problems at each iteration disregarding the fact that some other similar forward problems have been solved before. It is here where a well-trained surrogate model performs the best and makes the MCMC simulation much more efficient.

The basis of a FE procedure is the set of all $N_{i}(\boldsymbol{x})$ functions used to construct the solution $\boldsymbol{u}^{h}(\boldsymbol{x})$ as shown in (13) (and equivalently for pressure (14)). Note that each function $N_{i}(\boldsymbol{x})$ is associated with a node of the FE mesh and therefore the number of functions is usually very large. One key aspect of the functions $N_{i}(\boldsymbol{x})$ is their local character. This means that although the resulting matrices after discretization may be large, they are also sparse; a useful feature exploited by FE solvers.

The main idea of MOR techniques, and in particular of the RB approach, is to approximate the solution $\mathbf{u}$ (and that for $\mathbf{p}$ in mixed formulations) in terms of a smaller set of functions $\mathbf{b}_{m}$ as

$$
\mathbf{u} \approx \mathbf{u}_{\mathrm{RB}}=\sum_{m=1}^{n_{\mathrm{RB}}} \alpha_{m} \mathbf{b}_{m}
$$

where the basis $\left\{\mathbf{b}_{1}, \ldots \mathbf{b}_{n_{\mathrm{RB}}}\right\}$ is no longer associated with mesh nodes and thus no longer local and $\alpha_{m}$ are the unknowns. Therefore, for any new parameter (e.g. viscosity) input into (10), we seek a solution to (15) in a lower-dimension space $n_{\mathrm{RB}} \ll n_{\mathrm{FE}}$.

While simple in principle, this idea rises three important questions. First, how accurate is the reduced solution $\mathbf{u}_{\mathrm{RB}}$ compared to the high-fidelity $\mathrm{FE}$ solution $\mathbf{u}$ ? Second, how are the basis vectors $\mathbf{b}_{m}$ actually obtained? Third, given a set of basis vectors $\mathbf{b}_{m}$, how is the solution $\mathbf{u}_{\mathrm{RB}}$ computed? We answer these three questions below. For clarity, however, we will first describe the simpler problem of obtaining reduced solutions $\mathbf{u}_{\mathrm{RB}}$ assuming that we have an available set of basis vectors $\mathbf{b}_{m}$. 
The key idea behind the RB strategy is to create a basis of $n_{\mathrm{RB}}$ independent FE solutions $\left(\mathbf{u}_{m}, \mathbf{p}_{m}\right)$ of different realizations of the same problem (e.g. by varying the material properties). This group of solutions (sometimes called "snapshots") will be used as a basis. For the velocity, $\mathbf{b}_{m}=\mathbf{u}_{m}$, represents a subset of $\mathbb{R}^{3 n_{u}}$,

$$
\operatorname{span}\left\{\mathbf{u}_{1}, \mathbf{u}_{2}, \ldots, \mathbf{u}_{n_{\mathrm{RB}}}\right\} \subset \mathbb{R}^{3 n_{u}} .
$$

Similarly for pressure, the group of solutions $\mathbf{p}_{m}$ represents a subset of $\mathbb{R}^{n_{p}}$

$$
\operatorname{span}\left\{\mathbf{p}_{1}, \mathbf{p}_{2}, \ldots, \mathbf{p}_{n_{\mathrm{RB}}}\right\} \subset \mathbb{R}^{n_{p}}
$$

Every time a new problem has to be solved, its solution $\boldsymbol{\alpha}=\left[\alpha_{1}, \ldots, \alpha_{n_{\mathrm{RB}}}\right]^{\top}$, is sought in $\mathbb{R}^{n} \mathrm{RB}$ instead of in $\mathbb{R}^{n} \mathrm{FE}$ thus reducing dramatically the computational cost since $n_{\mathrm{RB}} \ll n_{\mathrm{FE}}$. The reduced solution is found as a linear combination of the elements of the basis

$$
\left[\begin{array}{l}
\mathbf{u} \\
\mathbf{p}
\end{array}\right] \approx\left[\begin{array}{l}
\mathbf{u}_{\mathrm{RB}} \\
\mathbf{p}_{\mathrm{RB}}
\end{array}\right]=\sum_{m=1}^{n_{\mathrm{RB}}} \alpha_{m}\left[\begin{array}{l}
\mathbf{u}_{m} \\
\mathbf{p}_{m}
\end{array}\right]=\left[\begin{array}{l}
\mathbf{B}_{\mathbf{u}} \\
\mathbf{B}_{\mathbf{p}}
\end{array}\right] \boldsymbol{\alpha}
$$

where $\boldsymbol{\alpha}$ is the vector of unknowns and $\mathbf{B}_{\mathbf{u}}=\left[\mathbf{u}_{1}, \ldots, \mathbf{u}_{n_{\mathrm{RB}}}\right]$ and $\mathbf{B}_{\mathbf{p}}=\left[\mathbf{p}_{1}, \ldots, \mathbf{p}_{n_{\mathrm{RB}}}\right]$ are matrices representing the reduced basis for velocity and pressure respectively in which each column corresponds to an FE solution. Note that in our implementation, two separated basis have been defined for velocity and pressure, but both share the same number of functions $n_{\mathrm{RB}}$.

The solution to the Stokes problem is obtained by replacing $\mathbf{u}$ and $\mathbf{p}$ with their reduced approximations from (19) into (15) and multiplying both sides by the basis to obtain,

$$
\left[\begin{array}{l}
\mathbf{B}_{\mathbf{u}} \\
\mathbf{B}_{\mathbf{p}}
\end{array}\right]^{\top}\left[\begin{array}{cc}
\mathbf{K} & \mathbf{G} \\
\mathbf{G}^{\top} & \mathbf{0}
\end{array}\right]\left[\begin{array}{l}
\mathbf{B}_{\mathbf{u}} \\
\mathbf{B}_{\mathbf{p}}
\end{array}\right] \boldsymbol{\alpha}=\left[\begin{array}{l}
\mathbf{B}_{\mathbf{u}} \\
\mathbf{B}_{\mathbf{p}}
\end{array}\right]^{\top}\left[\begin{array}{l}
\mathbf{f} \\
\mathbf{0}
\end{array}\right]
$$

Performing the multiplications, the system above is written as

$$
\left[\mathbf{B}_{\mathbf{u}}^{\top} \mathbf{K B}_{\mathbf{u}}+\mathbf{B}_{\mathbf{u}}^{\top} \mathbf{G B}_{\mathbf{p}}+\mathbf{B}_{\mathbf{p}}^{\top} \mathbf{G}^{\top} \mathbf{B}_{\mathbf{u}}\right] \boldsymbol{\alpha}=\mathbf{B}_{\mathbf{u}}^{\top} \mathbf{f} .
$$

Since all elements in $\mathbf{B}_{\mathbf{u}}$ are FE solutions, they all satisfy the incompressibility condition, namely $\mathbf{G}^{\top} \mathbf{u}_{m}=0$, for $m=1, \ldots, n_{\mathrm{RB}}$. Therefore,

$$
\mathbf{G}^{\top} \mathbf{B}_{\mathbf{u}}=0
$$

is always satisfied (independently of $\boldsymbol{\alpha}$ ) and the second and third terms in the left-hand side of equation (21) vanish. Therefore, the final reduced system of equations reads,

$$
\mathbb{K}_{\mathrm{RB}} \boldsymbol{\alpha}=\mathbb{E}_{\mathrm{RB}}
$$

where the reduced stiffness matrix and reduced force vector are $\mathbb{K}_{\mathrm{RB}}=\mathbf{B}_{\mathbf{u}}^{\top} \mathbf{K B}_{\mathbf{u}}$ and $\mathbb{F}_{\mathrm{RB}}=\mathbf{B}_{\mathbf{u}}^{\top} \mathbf{f}$, respectively. Note that $\mathbb{K}$ has the size of the reduced basis, $n_{\mathrm{RB}} \times n_{\mathrm{RB}}$, which is significantly smaller than the traditional FE nodal basis. For example, in the numerical examples presented below, $n_{\mathrm{RB}}$ is of the order of a few hundreds while the full $\mathrm{FE}$ basis is $>3 \times 10^{4}$, even for the smallest example.

When using iterative methods to solve the system XX, equation (22) is no longer satisfied. In this case, the terms including $\mathbf{G}$ in equation (21) do not strictly vanish and should be explicitly considered. However, the additional computational cost is insignificant, as $\mathbf{G}$ is much smaller than $\mathbf{K}$ and the terms including $\mathbf{G}$ change only when the bases are updated. 


\subsubsection{Error estimation}

The discrete space described by the reduced basis $\mathbb{R}^{n} \mathrm{RB}$ is an approximation of the FE discrete space $\mathbb{R}^{n} \mathrm{FE}$, and as such, the RB solution $\mathbf{u}_{\mathrm{RB}}$ has an associated error with respect to the high-fidelity $\mathrm{FE}$ solution $\mathbf{u}$. In reality, the FE solution is also an approximation of the real forward problem, albeit a better one. The estimation of the errors associated with FE solutions is a well-known procedure and it has been described in detail elsewhere (Zienkiewicz et al., 2005; Hughes, 1987). Here we will therefore focus on the estimation of the reduced basis solution with respect to the highfidelity FE one (instead of measuring the error with respect to the analytic solution). Specifically, we would like to measure how well the reduced basis performs, assuming that the best possible solution is that given by the FE solver.

Within this context, we define

$$
\mathbf{e}_{\mathbf{u}}:=\mathbf{u}-\mathbf{u}_{\mathrm{RB}}, \quad \mathbf{e}_{\mathbf{p}}:=\mathbf{p}-\mathbf{p}_{\mathrm{RB}}
$$

where $\mathbf{e}_{\mathbf{u}}$ and $\mathbf{e}_{\mathbf{p}}$ are the errors introduced by the RB solution with respect to the FE solution. The error equation associated with the RB solution is

$$
\left[\begin{array}{cc}
\mathbf{K} & \mathbf{G} \\
\mathbf{G}^{\top} & \mathbf{0}
\end{array}\right]\left[\begin{array}{l}
\mathbf{e}_{\mathbf{u}} \\
\mathbf{e}_{\mathbf{p}}
\end{array}\right]=\left[\begin{array}{c}
\mathbf{f} \\
\mathbf{0}
\end{array}\right]-\left[\begin{array}{cc}
\mathbf{K} & \mathbf{G} \\
\mathbf{G}^{\top} & \mathbf{0}
\end{array}\right]\left[\begin{array}{l}
\mathbf{u}_{\mathrm{RB}} \\
\mathbf{p}_{\mathrm{RB}}
\end{array}\right]
$$

Therefore, the residual associated with the $\mathrm{RB}$ solution reads,

$$
\mathbf{r}=\mathbf{f}-\mathbf{K} \mathbf{u}_{\mathrm{RB}}-\mathbf{G p}_{\mathrm{RB}}
$$

since due to the incompressibility condition in (22), the part of the residual corresponding to the pressure vanishes identically, $-\mathbf{G}^{\top} \mathbf{u}_{\mathrm{RB}}=0$. Note, however, that both the velocity and the pressure $\mathrm{RB}$ solutions contribute to the velocity part of the residual. It is usually a good estimator of the energy norm of the error (Quarteroni et al., 2016; Hesthaven et al., 2016). If the solution $\mathbf{u}_{\mathrm{RB}}$ and $\mathbf{p}_{\mathrm{RB}}$ were exact (i.e. equal to the $\mathrm{FE}$ solution), the residual would be zero, and so would the error. It follows that a large residual implies a large error in the RB approximation.

\subsubsection{Quantity of interest}

Instead of using the full residual in equation (26) as a measure of the error introduced by the RB approximation, here we introduce a more suitable goal-oriented criterion. The key idea is that such a criterion allows us to assign different weights to the error in different regions of the domain (regions of interest), and to control the maximum admissible error on any particular Quantity of Interest (QoI). The latter is typically an "important" model prediction that can be compared against data or that controls an important output of the model (Florentin \& Díez, 2012). Therefore, we do not seek to obtain accurate solutions for the entire numerical domain or for all model predictions, but rather to guarantee accurate RB solutions to the quantity or quantities that are of interest for the particular purpose of the inversion. This way of measuring the error of the RB approximation is key to maintaining the overall size of the RB small, as the basis is trained specifically to represent well (within admissible errors) only the relevant part of the solution, that is, the QoI.

There are two important points to clarify here. First, the RB approach discussed in this paper is independent of how the QoI is defined and it can accept other error measures. Second, that the accuracy of a global solution is not pursued does not mean that the approximation of the QoI will be of poor quality. On the contrary, we emphasize again that accurate solutions to the QoI are enforced and guaranteed by design. In the limiting case where the QoI is of "global" character (e.g. if the residual is used to estimate the error), the error will be a measure of accuracy for the entire 
domain and the resulting $\mathrm{RB}$ approximation will be trained to reproduce the solution in any region of the domain with the same accuracy.

The QoI is a scalar quantity $Q$, computed as a linear function, $l^{\circ}$, that extracts the relevant information from the global solution $\boldsymbol{u}$,

$$
Q=l^{\circ}(\boldsymbol{u}) .
$$

The discrete version of the linear QoI can be expressed as a product of one vector $\mathbf{f}^{o}$ describing the function and the solution $\mathbf{u}$ as,

$$
Q_{\mathrm{FE}}=\mathbf{f}^{o \top} \mathbf{u}
$$

In order to obtain an error representation based on the QoI, a dual (or adjoint) problem is typically defined (e.g. Florentin \& Díez, 2012). The weak form of the dual problem reads: find $(\boldsymbol{v}, d) \in \mathcal{V} \times \mathcal{L}_{2}(\Omega)$ such that,

$$
\left\{\begin{aligned}
a(\boldsymbol{w}, \boldsymbol{v})+b(\boldsymbol{w}, d) & =l^{o}(\boldsymbol{w}) & & \forall \boldsymbol{w} \in \mathcal{V} \\
b(\boldsymbol{v}, q) & =0 & & \forall q \in \mathcal{L}_{2}(\Omega)
\end{aligned}\right.
$$

This dual problem is discretized in the same FE spaces as the direct problem (15), which gives

$$
\left[\begin{array}{cc}
\mathbf{K} & \mathbf{G} \\
\mathbf{G}^{\top} & \mathbf{0}
\end{array}\right]^{\top}\left[\begin{array}{l}
\mathbf{v} \\
\mathbf{d}
\end{array}\right]=\left[\begin{array}{c}
\mathbf{f}^{o} \\
\mathbf{0}
\end{array}\right]
$$

For the Stokes problem discussed here, the stiffness matrix is symmetric and therefore the dual problem differs from the direct one only in the source term, which corresponds to the discretized linear operator representing the QoI.

Finally, using equations (15), (28) and (30), a representation of the error in the QoI associated with the RB approximation can be defined as,

$$
\begin{aligned}
E_{Q}=Q_{\mathrm{FE}}-Q_{\mathrm{RB}} & =\mathbf{f}^{o \top}\left(\mathbf{u}-\mathbf{u}_{\mathrm{RB}}\right) \\
& =[\mathbf{K} \mathbf{v}+\mathbf{G} \mathbf{d}]^{\top}\left(\mathbf{u}-\mathbf{u}_{\mathrm{RB}}\right) \\
& =\mathbf{v}^{\top} \mathbf{K}\left(\mathbf{u}-\mathbf{u}_{\mathrm{RB}}\right)+\mathbf{d}^{\top} \mathbf{G}^{\top}\left(\mathbf{u}-\mathbf{u}_{\mathrm{RB}}\right) \\
& =\mathbf{v}^{\top}\left(\mathbf{f}-\mathbf{K} \mathbf{u}_{\mathrm{RB}}\right)
\end{aligned}
$$

where the incompressibility condition in (22) is used and $\mathbf{v}^{\top} \mathbf{K u}=\mathbf{f}$ because $\mathbf{v}^{\top} \mathbf{G} \mathbf{p}=0$ (incompressibility of the dual problem). A residual-like vector is defined as,

$$
\mathbf{r}^{*}:=\mathbf{f}-\mathbf{K} \mathbf{u}_{\mathrm{RB}}
$$

and although different from the residual $\mathbf{r}$ in $(26), \mathbf{v}^{\top} \mathbf{r}^{*}=\mathbf{v}^{\top} \mathbf{r}$ holds because $\mathbf{v}^{\top} \mathbf{G} \mathbf{p}_{\mathrm{RB}}=$ 0 .

According to equation (31), the two ingredients needed to obtain a representation of the error are: i) the solution to the dual problem $\mathbf{v}$, and ii) the residual-like vector $\mathbf{r}^{*}$. A critical point here is that if the solution of the dual problem requires as much computational effort as the solution of the primal problem (the original Stokes problem), then the method becomes impractical. We can therefore replace the exact solution of the adjoint problem with an approximate (and much more efficient) solution, $\hat{\mathbf{v}}$, and define an error estimator for $E_{Q}$ as

$$
E_{Q} \approx \hat{E}_{Q}=\hat{\mathbf{v}}^{\top} \mathbf{r}^{*}
$$


We have tested two different approaches for computing $\hat{\mathbf{v}}$ and avoiding the explicit computation of the solution of the adjoint problem. The first and more computationally expensive option is to build a Reduced Basis for the adjoint problem. Instead of using a full FE solver, we build a basis for the adjoint using the same procedure we use for the primal problem. The error estimator of the solution of the adjoint is computed using the norm of the residual. The second and more efficient option is based on one important property of the adjoint problem: adjoints are typically less sensitive to parameters than the primal problem (Florentin \& Díez, 2012; García-Blanco et al., 2017; Serafin et al., 2017) and therefore the same adjoint solution can be used to estimate the error of many similar primal solutions. In the latter case, a single solution of the adjoint problem is required and then used to estimate the errors of all subsequent primal problems. An interesting variant of this case is to recompute the adjoint every $n$ number of primal solutions to guarantee that its solution remains relevant for the values that are being tested. Numerous tests demonstrated that the second option without recomputing the adjoint is the optimal one from a cost-accuracy point of view. We do not claim, however, that this strategy applies to other forward problems; verification tests should be performed for different problems.

\subsubsection{Building the reduced basis $\mathrm{B}_{\mathrm{u}}$}

Typical MOR implementations consist of two stages: 1) the offline stage, where all costly computations (involving a high-fidelity solver) dependent on $\mathcal{V}^{h}$ are performed; and 2) the online stage, where the reduced order solver or "surrogate" is used to obtain fast and computationally inexpensive solutions independent of $\mathcal{V}^{h}$ (Quarteroni et al., 2016; Hesthaven et al., 2016; Ito \& Ravindran, 1998; Prud'homme et al., 2002). While in principle a similar splitting can be used in the context of a probabilistic inverse problem (Galbally et al., 2009; Wang \& Zabaras, 2005; Lieberman et al., 2010), it is not always straightforward to predict which FE solutions (or snapshots) should be computed a priori during the offline stage, as there is no way of knowing in advance which solutions are most relevant as the MCMC converges towards a reduced parameter space (i.e. the high probability regions). As the number of parameters increases, predicting the relevant high probability regions becomes more difficult. For this reason, here we prefer to combine the offline and online stages into a single stage where the reduced basis is created on the fly and as needed during the inversion processes.

In this greedy approach, the basis $\mathbf{B}_{\mathbf{u}}$ is initially empty (but not necessarily) and is subsequently enriched with FE (high-fidelity) solutions as the inversion progresses. The first forward problem required by the inversion is solved with the high-fidelity FE solver and its solution is added to the basis. From then on, every time a new forward problem needs to be solved, the solution is sought in the RB space $\mathbb{R}^{{ }^{n} \mathrm{RB}}$ and the error of the RB approximation is assessed. If the error is larger than a predefined tolerance, $e_{\mathrm{RB}}$, the problem is solved using $\mathrm{FE}$ and the new high-fidelity solution is added to $\mathbf{B}_{\mathbf{u}}$ (i.e. the basis is enriched). Therefore, the basis is only enriched when the accuracy of the RB solution is below the specified tolerance. It minimizes the possibility of having a basis with strong internal correlations (nearly linear dependant, see below).

Since a MCMC-based inversion gradually converges to an equilibrium distribution that typically spans only a limited region of the initial parameter space, it is expected that the basis will initially grow until it becomes rich enough to provide accurate approximations (within a tolerance) to any subsequent forward evaluation. In this way, the basis does not need to provide accurate solutions for the entire parameter space, but it is automatically tailored to provide accurate solutions within the region of high probability as sampled by the MCMC. In all the numerical examples presented below, the number of FE evaluations needed to "saturate" the basis is a very small fraction of the total number of forward evaluations, making the RB approach 
extremely efficient. In other words, the converging nature of a MCMC-based inversion makes the option of RB solvers extremely attractive, as it is a situation where RB performs at its best.

The following flowchart (Figure 1) shows the building process of a reduced basis coupled simultaneously within an inversion framework.

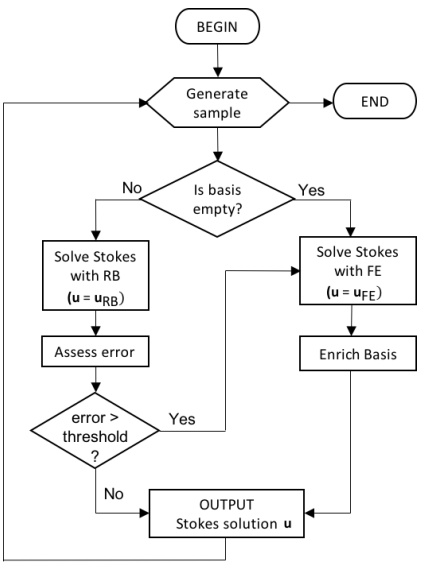

Figure 1. Flowchart of the greedy approach to build a Reduced Basis within an outer loop application (inversion problem)

Moreover, as the Markov chain moves to a more restricted parameter space, the space $\mathbb{R}^{n} \mathrm{RB}$ can be inspected at regular intervals and processed to remove bases that are not contributing significant information. The result is a more compact and datadriven basis tailored for the specific problem at hand. A somewhat similar strategy was used in Bui-Thanh et al. (2008), Florentin and Díez (2012), Lieberman et al. (2010), and Cui et al. (2014). The slight drawback of this approach is that instead of relaying on fast RB solutions since the beginning of the inversion, some computational cost must be spent in creating the basis on the fly. In most practical cases, however, this is not a problem. The overall cost involved in computing the basis during the inversion process is significantly less than that invested in creating sufficiently accurate basis in an offline stage, except when the parameter space is relatively small (an uncommon case in geophysical inversions). In addition, the basis created in one inversion can always be reused in subsequent inversions of similar characteristics.

Finally, the proposed greedy approach to build the RB does not guarantee orthogonality between the members of the basis. Redundant (linearly dependent) basis elements may still be included in the basis, which would result in an ill-conditioned linear system to be solved (23). In practice, however, we have not observed this behaviour. The condition number of the reduced system in our problems remains almost constant as the basis grows, indicating that the basis is not deteriorated by the greedy character of the algorithm. In fact, by construction, solutions which are linearly dependent (according to a predefined tolerance) should not have been added to the basis. Nevertheless, in the case of having redundant information in the basis, a Singular Value Decomposition procedure (Afonso et al., 2015) can be applied on-the-fly to obtain an orthogonal basis. Other possibilities include orthogonalizing the reduced basis every time a new snapshot is added, as in Lieberman et al. (2010), Cui et al. (2014), and Bui-Thanh et al. (2008). 


\section{Preliminary numerical considerations}

Before presenting a series of illustrative examples, we will first outline their basic setup and briefly discuss several modelling decisions and assumptions relevant for the application of the proposed method.

\subsection{Model discretization and parameterization}

We start by noting that the proposed methodology is independent of the chosen discretization/element type. In this work, all problems are discretized with TaylorHood hexahedral elements of order two for velocity and order one for pressure (Q2P1). Free slip boundary conditions are applied on the boundaries of the domain, i.e. the normal velocity is zero and the two tangential components do not change across the boundary (zero shear strain rates and stresses along the boundary). This discretization generates a numerical domain that can be seen as a collection of nonoverlapping adjacent rectangular columns (Figure 2).

We assign a unique model parameter to each of these columns, viz. lithospheric thickness, or equivalently, depth to the Lithosphere-Asthenosphere Boundary (LAB). Therefore, our vector of model parameters (to be retrieved by the inversion) is composed of the LAB depths for all columns in the numerical domain.

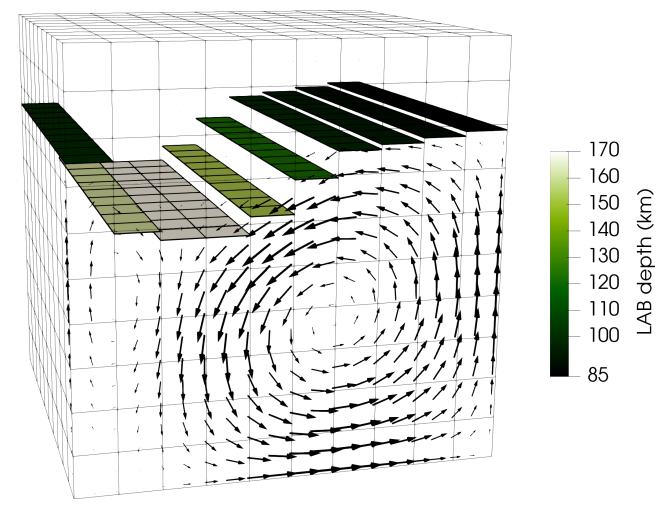

Figure 2. Idea of the inversion problem. Black arrows showing direction and magnitude of velocity field (observables). LAB depth discretized in 100 parameters (unknowns).

The LAB depth is the main parameter controlling the flow pattern inside the numerical box as it defines the internal temperature distribution, which in turn controls the viscosity and internal buoyancy forces. For illustrative purposes, the temperature inside each column is assumed to follow a linear profile defined by the temperature at the surface $\left(T_{0}=293 \mathrm{~K}\right)$ and that at the LAB depth $\left(T_{\mathrm{LAB}}=1523 \mathrm{~K}\right)$. In the asthenosphere, we also assume a constant linear gradient between $T_{\mathrm{LAB}}$ and a fixed bottom temperature $\left(T_{660}=1873 \mathrm{~K}\right)$. Absolute pressure is assumed to follow a lithostatic profile, $p=\rho_{0} g z$, where $g$ and $z$ are gravity and depth, respectively.

Density is computed as

$$
\rho=\rho_{0}\left(1-\alpha\left(T-T_{0}\right)+\beta\left(p-p_{0}\right)\right)
$$

where $\rho_{0}$ is the reference density at $T_{0}$ and $p_{0}$ and $\alpha$ and $\beta$ are the coefficients of thermal expansion and compressibility, respectively. 
Viscosity follows a power-law of the form,

$$
\mu=A_{D}^{-1 / n} \dot{e}_{\mathrm{II}}^{(1 / n)-1} \exp \left(\frac{E+p V}{n R T}\right)
$$

where $E$ is the activation energy, $V$ the activation volume, $R$ the universal gas constant, $n$ the flow law exponent, $\dot{e}_{\mathrm{II}}$ the second invariant of the strain rate and $A_{D}$ a constant depending on the material. As usual in geodynamic simulations, a threshold (maximum) viscosity, $\mu_{\max }$, is imposed. All values used are listed in Table 1 . Because the methodology presented here is suited for linear problems we linearize the Stokes equations by assuming a constant strain rate $\dot{e}_{\mathrm{II}}=10^{-15} \mathrm{~s}^{-1}$. We note, however, that the methodology can be adapted to nonlinear problems and it is independent of the specific parametrization used here.

Table 1. Values of the parameters used to compute material properties

\begin{tabular}{ccl} 
Parameter & Value & Units \\
\hline$\rho_{0}$ & 3300 & $\mathrm{~kg} \cdot \mathrm{m}^{-3}$ \\
$p_{0}$ & 0 & $\mathrm{MPa}$ \\
$T_{0}$ & 293 & $\mathrm{~K}$ \\
$T_{\mathrm{LAB}}$ & 1523 & $\mathrm{~K}$ \\
$T_{660}$ & 1873 & $\mathrm{~K}$ \\
$\alpha$ & $10^{-5}$ & $\mathrm{~K}^{-1}$ \\
$\beta$ & $10^{-5}$ & $\mathrm{MPa}^{-1}$ \\
$\mu_{\max }$ & $10^{24}$ & $\mathrm{~Pa} \cdot \mathrm{s}$ \\
$A_{D}$ & $1.1 \cdot 10^{5}$ & $\mathrm{MPa}{ }^{-n} \cdot \mathrm{s}^{-1}$ \\
$\dot{e}_{\mathrm{II}}$ & $10^{-15}$ & $\mathrm{~s}-1$ \\
$E$ & $5.3 \cdot 10^{5}$ & $\mathrm{~J} \cdot \mathrm{mol}^{-1}$ \\
$V$ & 14 & $\mathrm{~J} \cdot \mathrm{MPa}^{-1} \cdot \mathrm{mol}^{-1}$ \\
$R$ & 8.314 & $\mathrm{~J} \cdot \mathrm{mol}^{-1} \cdot \mathrm{K}^{-1}$ \\
$n$ & 3.5 & \\
\hline
\end{tabular}

\subsection{Synthetic observables}

The synthetic measurements or observations used in our examples are vertical mantle velocities at specific points within a predefined observation region. In reality, mantle velocities cannot be directly observed and other indirect observables such as timedependent topography (e.g. from GPS measurements) or plate motions need to be used. However, a number of additional assumptions and secondary quantities need to be considered in order to obtain these observables from the forward problem. Given the purpose of this work, we prefer to avoid this additional complexity and focus on the intrinsic features and performance of the presented method by using direct outputs of the forward problem only (i.e. velocities). In doing so, we can better isolate and analyse the main RB effects within the MCMC simulation in these numerical examples.

We create a dataset of synthetic observables to be used in our MCMC simulations from a high-resolution reference or "true" model (see Figure 3a in Section 5.1). The vertical components of the velocity field are computed at $n_{d}$ points and define the dataset of synthetic observables, $\mathbf{d}^{\text {obs }}$. Gaussian noise, $r \sim \mathrm{N}\left(0, \sigma_{d}\right)$, is used to introduce some controlled error in the synthetic observables. The standard deviation of the data errors, $\sigma_{d}$, is chosen to be a percentage of the maximum absolute value in the dataset $\mathbf{d}^{\text {obs }}$,

$$
\sigma_{d}=\sigma_{e} \max \left|\mathbf{d}^{\mathrm{obs}}\right| .
$$


Since here we are interested in studying the performance of the RB+MCMC method rather than the actual solution to the inverse problem, the following examples include the error in the data only in the likelihood function; i.e. no noise is actually added to the synthetic data. The reason for this choice is that when perturbations are added to the synthetic data, the posterior PDFs will not necessarily converge to the reference model, but to a "perturbed" model that best fits the contaminated observables, thus complicating the performance analysis of the method. However, in order to show that the $\mathrm{MCMC}+\mathrm{RB}$ also works in the case of contaminated data, a second version of the first example of Section 5.1, including perturbations to the observables, is shown in the supplementary material.

The observation region $\Gamma$ is defined in accordance with the a priori information on the parameters used in the MCMC simulation (Section 4.3). This implies that parameters are sampled within $\Gamma$, and therefore the largest changes in the velocity field are observed in this region. Moreover, we want the vertical components of velocity to be accurately computed in this region, so we make $\Gamma$ coincide with the region of interest used to compute the QoI, which controls the accuracy of the RB solution (See Sections 3.2.3 and 4.4). The number of data points will be specified for each example and will vary depending on the number of unknown parameters in the inversion.

\subsection{Sampling strategy and Likelihood function}

In order to test the MCMC+RB method in adverse conditions, a relatively uninformative initial scenario is defined. The prior PDF for all parameters in each column are set as uniform within a range of $100 \mathrm{~km}$ (i.e. $\pm 50 \mathrm{~km}$ from the reference LAB value). With these priors, all gain of information, as contained in the posterior PDF, will be controlled exclusively by the likelihood. This allows a readily interpretation of the results.

The prior PDF is sampled using a multivariate Gaussian proposal distribution centered at the current state of the chain $\mathbf{m}_{\text {old }}$,

$$
\mathbf{m}_{\text {new }} \sim \mathrm{N}\left(\mathbf{m}_{\text {old }}, \sigma_{p}^{2}\right) .
$$

For each proposed move $\mathbf{m}_{\text {new }}$, only one parameter $m_{i}, i \in\left[1, n_{m}\right]$, is modified with respect to $\mathbf{m}_{\text {old }}$. In other words, we modify the LAB structure by changing only one column per iteration according to equation (37). The chosen value for $\sigma_{p}$ will be specified for each example.

For the likelihood function (see equation (4)), we choose a standard Gaussian (L-2 norm) form

$$
L(\mathbf{m})=\frac{1}{2 \sigma_{d}^{2}} \sum_{i \in \Gamma}\left(u_{z i}(\mathbf{m})-d_{i}^{\mathrm{obs}}\right)^{2}
$$

where $i$ ranges from 1 to the number of points $n_{d}$ in the region of interest $\Gamma$ and $d_{i}^{\text {obs }}$ and $u_{z i}(\mathbf{m})$ are the "observed" (synthetic) and predicted vertical velocities at point $i$, respectively. The standard deviation $\sigma_{d}$ is related to the error in the measurements and is defined in equation (36). We also apply a multiplicative factor to $\sigma_{d}$ to account, as a first-order correction, for modelling/theoretical uncertainties. In doing so, we always keep the error of the RB one order of magnitude smaller than the above uncertainty (more details in Section 5.1.2). We acknowledge that other strategies to account for modelling uncertainties in Bayesian inversions could also be considered (see Linde et al. (2017) for a review), for instance, via a full covariance matrix defining model errors (see also a discussion in Afonso et al., 2013a,b). Another option could be to assign priors to these errors and let them be modelled as part of a hierarchical Bayesian inversion (Titus et al., 2017; Malinverno \& Briggs, 2004). 


\subsection{Reduced Basis solver}

As detailed in Section 3.2.3, we define a goal-oriented measure of the RB error, that is, we quantify the errors in some particular region of interest and for some particular QoI (27). We recall that this approach helps in keeping the overall size of the basis small. In the following examples, we define a region of interest $\Gamma$ as the volume enclosed by the a priori information on the model parameters: the 100km thick "layer" centered along the reference LAB. This volume naturally coincides with the information given to the MCMC solver; i.e. we want to reproduce the results accurately in a region where the model parameters will have the largest influence.

With the region $\Gamma$ defined, we proceed to define a QoI. This parameter summarizes the important part of the solution into one scalar number, so it is typically an integrated quantity. A possible definition of QoI is the integral of the vertical velocity within the region of interest $\Gamma$. However, for the incompressible Stokes problem, this integral vanishes identically for some configurations (e.g. a horizontal LAB). This is an undesired property, as the estimated error might be zero independently of the solution provided by the basis. Therefore, considering future (real) inversions where surface elevation and GPS velocities will be used as observables, it is more appropriate to define an error estimator that measures the gradient of the vertical velocity along the vertical direction (as this is a quantity from which dynamic topography effects can be estimated). The approach implicitly assumed is based on the rationale presented in (Afonso, Rawlinson, et al., 2016), among others, who decoupled lithospheric and sublithospheric (truly dynamic effect) contributions to surface elevation. In this approach, the region above the LAB is assumed to be cold enough to be considered essentially rigid and therefore its "static" contribution to surface elevation is accounted for by a simple lithospheric isostatic balance. Superimposed on this static contribution is the dynamic contribution from sublithospheric convection, which affects elevation via normal stresses associated with viscous flow and transferred to the lithosphere via viscous coupling in the lower regions of the lithosphere. With this in mind, we define the QoI as,

$$
Q=\int_{\Gamma} \frac{\partial u_{z}}{\partial z} d \Omega
$$

The QoI allows us to define the adjoint problem to get a representation of the error via equation (31). According to the discussion in Section 3.2.3, we solve the adjoint problem once at the beginning of the MCMC simulation and reuse its solution for assessing the error in all subsequent iterations.

At each inversion step, the matrix $\mathbb{K}_{\mathrm{RB}}$ (and $\mathbb{T}_{\mathrm{RB}}$ ) needs to be evaluated, since values of viscosity (and density) change. Performing the multiplication $\mathbf{B}_{\mathbf{u}}^{\top} \mathbf{K} \mathbf{B}_{\mathbf{u}}$ at each step can be time-consuming for very large bases. Our strategy takes advantage of the chosen LAB discretization and sampling strategy, i.e. not all columns of finite elements change their viscosities between successive inversion steps, but only a few. Taking this into account, we update $\mathbb{K}_{\mathrm{RB}}$ by just updating the contribution of the finite elements that have been modified at the current step. When the basis needs to be enriched, either the whole multiplication is performed or a new row and column are added to $\mathbb{K}_{\mathrm{RB}}$ to include the information of the new basis element. When the basis is rich enough and $\mathrm{FE}$ solutions are no longer required, the matrix $\mathbb{K}_{\mathrm{RB}}$ can be reused and updated at a very low cost for the rest of the MCMC inversion.

Not only this strategy is much faster, but it also allows us to avoid evaluating $\mathbf{K}$ at each inversion step. When needed, matrix $\mathbf{K}$ is also assembled following the same idea. The first time it is assembled accounting for the contribution of all finite elements in the mesh, whereas in the successive inversion steps, it is efficiently updated with just the contribution of the elements that changed. 


\section{Numerical examples}

In this section we present several inversion examples with the main goal of analyzing the interaction between MCMC and RB methods and their joint performance. We will show that MCMC together with $\mathrm{RB}$ reduces dramatically the cost of solving the inverse (geodynamic) problem. In all tested cases, the computational gain is an outstanding $\sim 99 \%$. In other words, our combined MCMC $+\mathrm{RB}$ strategy requires only $1 \%$ of the computational cost that would be needed in a standard MCMC inversion. We also note here that none of the examples below have used any parallelization technique to improve the efficiency of the forward problem; all were run in single CPU machines.

Two different types of illustrative examples are presented next. First, a set of simple examples of small size will be used to compare solutions obtained with our $\mathrm{MCMC}+\mathrm{RB}$ method versus those given by a standard $\mathrm{MCMC}+\mathrm{FE}$ approach as well as to analyze MCMC convergence, RB solver efficiency, and basis-size behavior. Second, a larger and more realistic example is used to illustrate the scalability and efficiency of the method.

\subsection{Simple 3D Cartesian model}

A 3D Cartesian domain representing a portion of the Earth from the surface down to $660 \mathrm{~km}$ depth is defined and discretized with $10 \times 10 \times 20$ finite elements. The LAB shape to be recovered (Figure 3a) is described along the examples with both 25 and 100 parameters. This setup is common to all the examples in this section unless stated otherwise. Regarding the number of synthetic observables, we fixed 675 points whitin the observation region $( \pm 50 \mathrm{~km}$ from the reference $\mathrm{LAB}$ value).

The initial model (starting point of the Markov chain) has a constant LAB at $130 \mathrm{~km}$ depth, except in one column, where the LAB is at $100 \mathrm{~km}$ depth (Figure $3 \mathrm{~b}$ ). This is simply to avoid an initial problem with zero velocity field.

a)

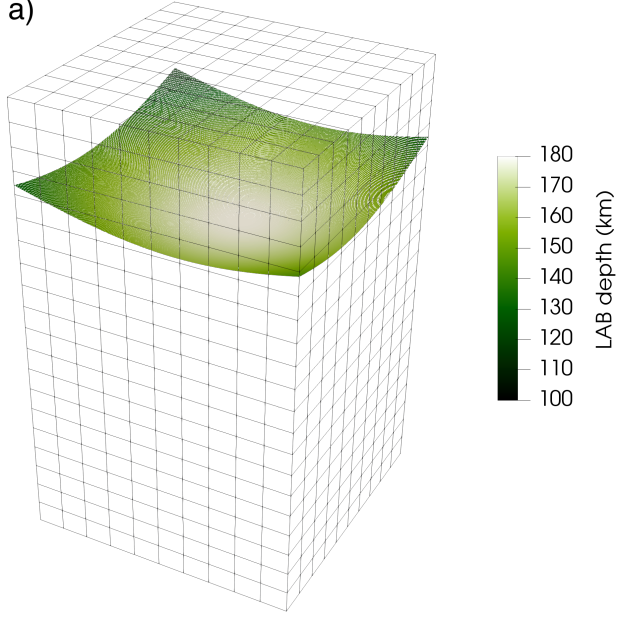

b)

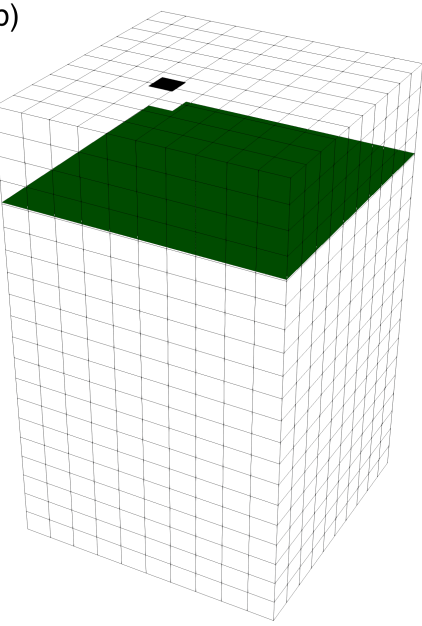

Figure 3. 3D cartesian domain discretized in 10x10x20 mixed elements representing a portion of Earth of $660 \mathrm{~km}$ depth and $400 \mathrm{~km}$ in the two directions along the surface. (a) Synthetic reference $\mathrm{LAB}$ and (b) initial model configuration. 


\subsubsection{MCMC convergence}

We run a total of $2 \times 10^{5}$ steps in the MCMC simulation. The first $10^{4}$ values considered burn-in and thus discarded for later analyses. The standard deviation of the proposal distribution in (37) is set at $\sigma_{p}=5 \mathrm{~km}$ and the percentage of the introduced noise in (36) is $\sigma_{e}=10^{-1}$. Regarding the Reduced Basis, a threshold $e_{\mathrm{RB}}=10^{-2}$ is set for the relative version of the error estimator in (33), which will control the accuracy of the RB solution.

The results of this inversion are summarized in Figures 4 and 5. Despite the uninformative initial scenario (uniform priors and flat initial state), these results demonstrate that the $\mathrm{MCMC}+\mathrm{RB}$ inversion algorithm succeeded in obtaining an accurate representation of the true PDF for all parameters. The trace plots in Figure 4 also show that the inversion converged quickly to the true posterior PDF (LAB values + their associated uncertainties). The posterior distributions for each parameter (Figure 5 ) exhibit mean values which coincide well with the reference synthetic LAB depths; all within one standard deviation (additional results can be found in the supplementary material).

Given the knowledge that we have over the true parameter space and uncertainties in these synthetic examples, both the burn-in period and the length of the simulations (as a convergence criteria) are chosen by visual inspection of the chains during the simulation.

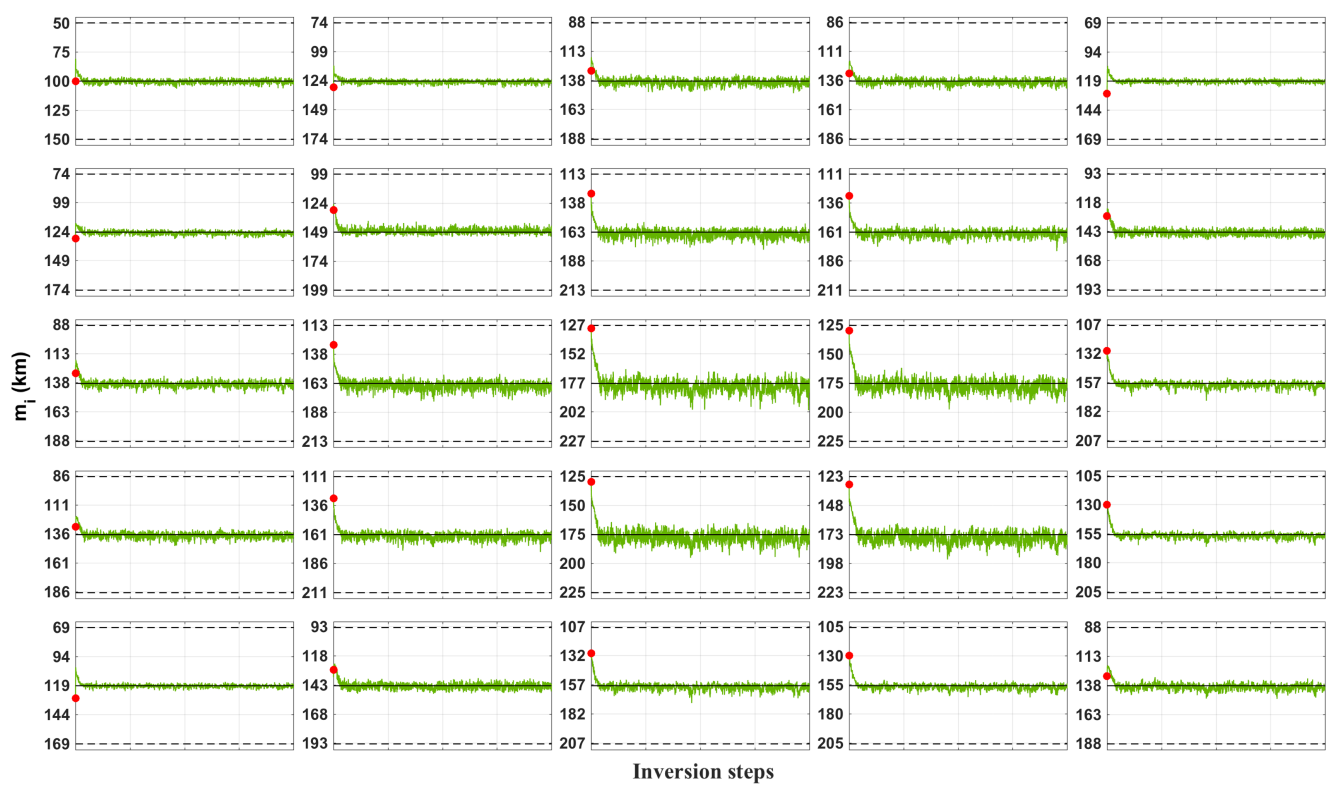

Figure 4. Random walk for each of the 25 parameters. In each plot: prior distribution (black dashed line), initial value (red dot), synthetic LAB value used to generate the perturbed velocity field (solid black line) and random walk of values of the posterior distribution (green). No burnin period has been discarded in this figure, so the initial value for each of the model parameters can be seen. 


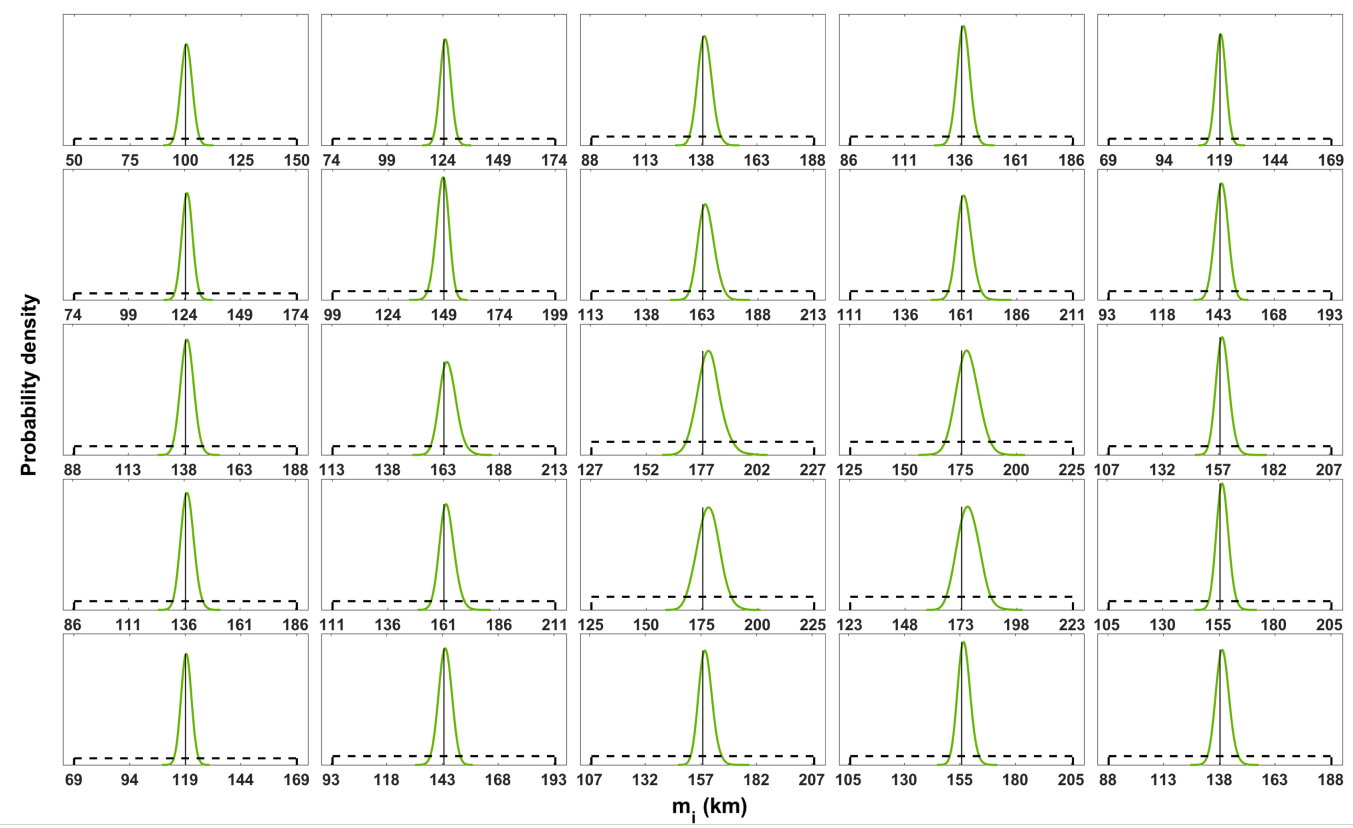

Figure 5. Posterior PDF for each of the 25 parameters. In each plot: prior distribution (black dashed line), synthetic LAB value used to generate the perturbed velocity field (solid black line) and PDF of the posterior distribution (green). A burn-in period has been discarded in the estimation of the posterior PDF for each of the model parameters.

\subsubsection{Reduced Basis solver efficiency}

For the present example, our algorithm required the solution of only $118 \mathrm{FE}$ problems to complete $2 \times 10^{5} \mathrm{MCMC}$ inversion steps, i.e. a $0.06 \%$. A FE solution takes around 6.3 seconds whereas the time for a RB solution with a basis of around 120 elements and updating between 80 and 160 finite elements ( 4 to 8 columns) at a time is on average 0.09 seconds. This constitutes a staggering gain in efficiency, since the $\mathrm{CPU}$ time of using the MCMC+RB strategy is only $1.5 \%$ of the (prohibitively large) time that would be required for the full $\mathrm{MCMC}+\mathrm{FE}$ option (i.e. one $\mathrm{FE}$ solution per MCMC step). Although the metric in this figure is not actually comparing CPU time, and it may give the impression that the RB solution is obtained at zero cost, the small $\mathrm{RB}$ times show that the real cost is little significant compared to the $\mathrm{FE}$ and, therefore, the metric used can be trusted.

The evolution of the basis size as a function of the first $10^{5}$ inversion steps is shown in Figure 6 (left axis). The red dashed line represents the cost of an inversion using FE only (no reduced basis). In the same figure we include the trace plot (right axis) for a representative parameter, showing rapid convergence and clear stationarity of the chain.

The behaviour shown in Figure 6 illustrates the key benefit of the coupled $\mathrm{MCMC}+\mathrm{RB}$ scheme, viz. the flattening of the basis size curve means that most inversion steps are RB solutions rather than full FE solutions. Once the basis is rich enough to approximate well forward problems within the high-probability space, its size stops increasing. Moreover, the longer the MCMC simulation the most favourable/efficient the RB strategy becomes, since all solutions will be required with RB. 


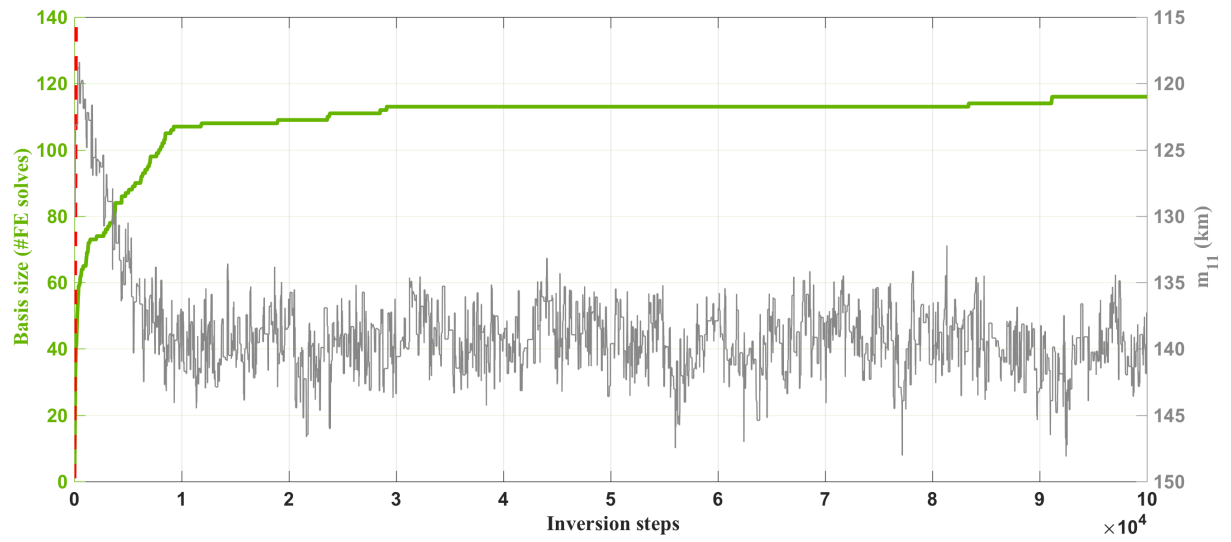

Figure 6. Differences between the number of full FE problems solved during the inversion using the RB approach (green solid line) and using standard FE (red dashed line). Random walk for a parameter (gray). The flattening of the basis size curve shows that most inversion steps are being solved without the need to solve the full FE problem. Once the basis is rich enough to represent the space tested by the inverse solver, its size stops increasing as any solution is properly addressed by the RB and no new full FE solver are required.

During the initial steps of the inversion, the high probability regions of the parameter space are still poorly known and large changes in the parameter space exploration are expected. This, together with the fact that the basis is very small, makes that the initial number of $\mathrm{FE}$ solves required is relatively large. As the random walk converges, the size of the basis stabilizes.

The use of RB instead of FE introduces an error in the solution of each forward problem. This error is controlled by the predefined tolerance for the RB. To test that the behaviour of the MCMC simulation is not affected by this error to any significant extent, we run three identical MCMC inversions: two of them using a MCMC+RB approach with different errors $e_{\mathrm{RB}}=10^{-3}$ and $10^{-2}$, and one using full $\mathrm{FE}$ solutions at every step of the chain. Since the previous example showed that the chain converges quickly, the number of inversion steps performed for this test was reduced to $10^{5}$, as otherwise the cost of the FE strategy would render the inversion impractical.

Figure 7 compares the results from these 3 strategies for the same model parameter (all other parameters behave in a similar way and therefore we do not plot them here). These results clearly demonstrate that all MCMC simulations are in excellent agreement. The MCMC+RB strategy is not affected by the RB error and performs properly as a surrogate of a FE forward model both in terms of accuracy, and particularly, in efficiency. The basis size strongly depends on the error assigned to the RB, $e_{\mathrm{RB}}$. In the examples shown here, the basis sizes are $\sim 374 \mathrm{FE}$ and $\sim 109 \mathrm{FE}$ for errors of $10^{-3}$ and $10^{-2}$, respectively. According to these values, and with the idea of keeping the basis as small as possible (within the limits of the observables error), we decided to use $e_{\mathrm{RB}}=10^{-2}$ for the remaining examples.

Another important point to remark about the efficiency of the MCMC-RB strategy is that the number of elements in the basis does not depend on the discretization of the forward solver. In other words, for a fixed number of parameters and guaranteeing a minimum accuracy according to the problem at hand, a higher discretization of the mesh does not affect the number of elements in the basis. To illustrate this, the same inversion was run five times for the same number of parameters, but varying 


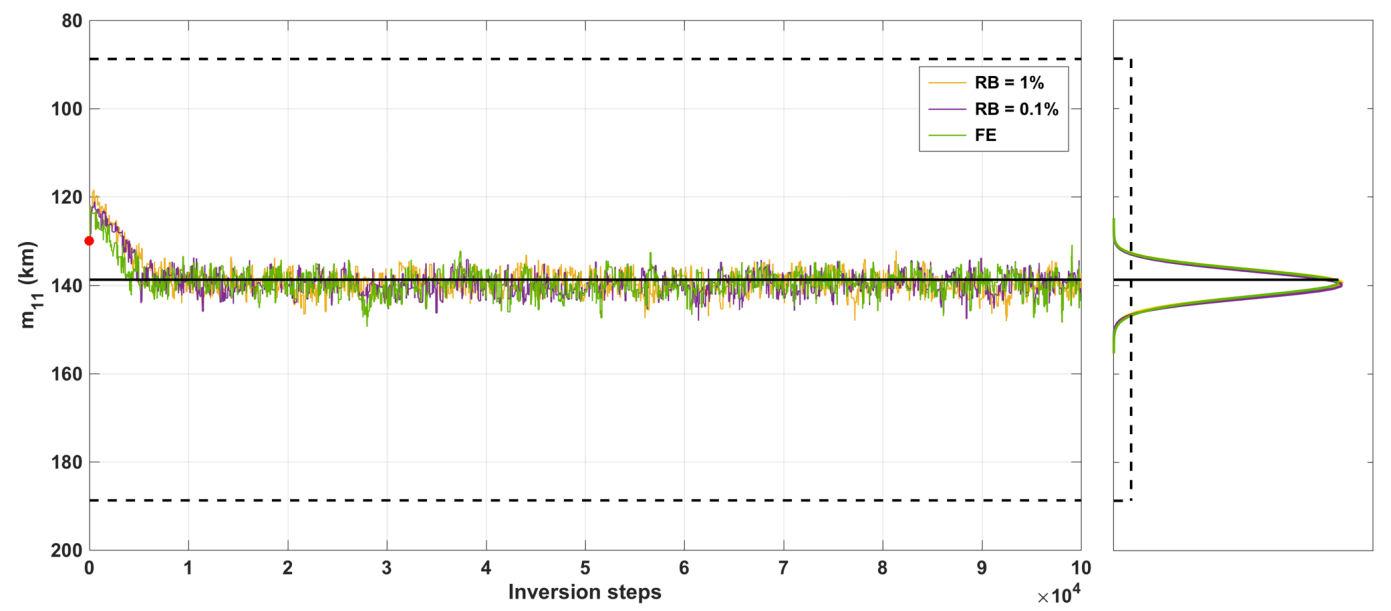

Figure 7. Differences between RB approach (green) and standard FE (red) in the random walk and posterior PDF for one parameter. In each plot: prior distribution (black dashed line), initial value (red dot) and reference synthetic LAB depth (black solid line).

the discretization of the mesh of the forward problem. The FE direct solver used here and the available computational resources allowed us to solve the Stokes problem with meshes up to $32000 \mathrm{FE}$ (Q2-P1). Table 2 shows the corresponding final basis sizes. In all cases, the final basis sizes are comparable, showing no correlation with mesh size.

Table 2. Final basis size obtained during the inversion of 25 parameters and $2 \times 10^{5}$ inversion steps using different mesh sizes for the solution of the forward problem

\begin{tabular}{c|c} 
Mesh size & Basis size \\
\hline 2000 & 118 \\
4500 & 115 \\
8000 & 119 \\
18000 & 104 \\
32000 & 108 \\
\hline
\end{tabular}

Although the number of elements in the basis does not change, it is important to clarify that each element of the basis has the length of the solution of the forward problem. Therefore, a very fine discretization certainly contributes to increase the overall size of the basis. However, as disscused in Section 4.4 the multiplication $\mathbf{B}_{\mathbf{u}}^{\top} \mathbf{K B}_{\mathbf{u}}$ is not actually performed and, therefore, the length of the basis elements is not a problem for the MCMC-RB strategy.

These results show that both the number of elements in the basis and the strategy to update $\mathbb{K}_{\mathrm{RB}}$ do not depend on the discretization of the forward problem, and therefore, the limiting factor determining the efficiency of the method is not the MCMC+RB method but the actual FE solver itself.

\subsubsection{A larger parameter space}

The following example illustrates the effect that a larger number of model parameters has in the size of the generated basis. Intuitively, it is expected that the size of the 
basis would be proportional to the size of the parameter space. This is because more parameters allow to describe a richer family of solutions and, therefore, the amount of information required to span all possible solutions grows as well. In the present example, we show that although the the size of the basis grows with the number of parameters, the method remains practical. The LAB structure is now discretized with 100 parameters and the number of synthetic observables is increased to 2700 to make the overall sensitivity comparable to that in the previous example. The setup of the MCMC simulation is identical to that of the previous example (same priors and starting configuration, $10^{6}$ steps, $\sigma_{p}=5 \mathrm{~km}, e_{\mathrm{RB}}=10^{-2}$ ).

The basis needed about 262 elements ( $0.03 \%$ of the total) to create an accurate surrogate for all simulation steps, which again represents a drastic reduction, an approximately $0.8 \%$ of the overall CPU time if only using FE. In this example the time of a RB solution is around 0.05 seconds since the number of columns modified per inversion step is smaller (one FE column per parameter). Figure 8 summarizes the posterior PDF for LAB structure as the mean of each of the parameters.

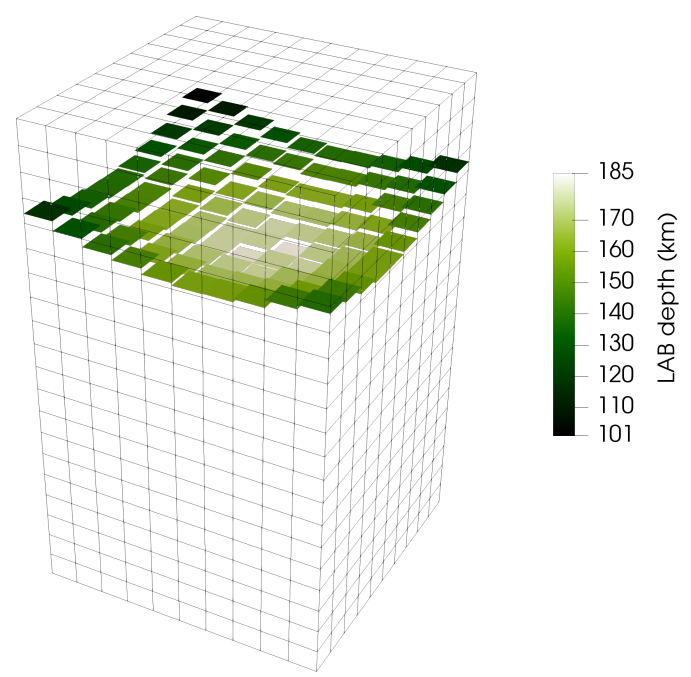

Figure 8. Mean values of the posterior PDFs for each of the unknown parameters obtained from solving the inverse problem. Unknown LAB discretized 100 parameters.

The algorithm clearly succeeded in obtaining an accurate representation of the solution. Differences between mean values of the posterior PDF and the reference synthetic LAB are again within one standard deviation. Plots of the random walks and posteriors for each of the 100 parameters and values of means and standard deviations can be found in the supplementary material. The standard deviations of the posteriors range approximately between $2 \mathrm{~km}$ and $6 \mathrm{~km}$. These values are very close to the ones obtained in the previous example. This is to be expected, since we increased the number of observables to make the overall sensitivity comparable in both examples. If the number of data points is kept fixed, the posterior distributions become wider as the number of parameters increase (i.e. as the discretization is made finer). This suggests that the sensitivity of the velocity field to perturbations of a single parameter is reduced as the number of parameters increases (i.e. finer discretization). This behaviour is wellknown in inverse problems, where a parsimonious trade-off always exists between the number of parameters needed to represent a model versus the number that can be well-constrained by the data. Less sensitivity to model parameters requires exploring larger areas of the parameter space, which typically results in longer chains with wider posteriors. 
This example also illustrates the scalability of the method. Figure 9 shows the evolution of the basis for different number of parameters: 25, 100 and 225. The number of synthetic observables is increased proportionally so that sensitivity is not a controlling factor: 675,2700 and 6075 data points respectively. In all cases, the growth of the basis (i.e. the number of full FE problems) reaches a "plateau" or a "saturation" level after which all MCMC steps are computed with the RB surrogate. This behaviour can be exploited to create even more efficient algorithms (see next section).

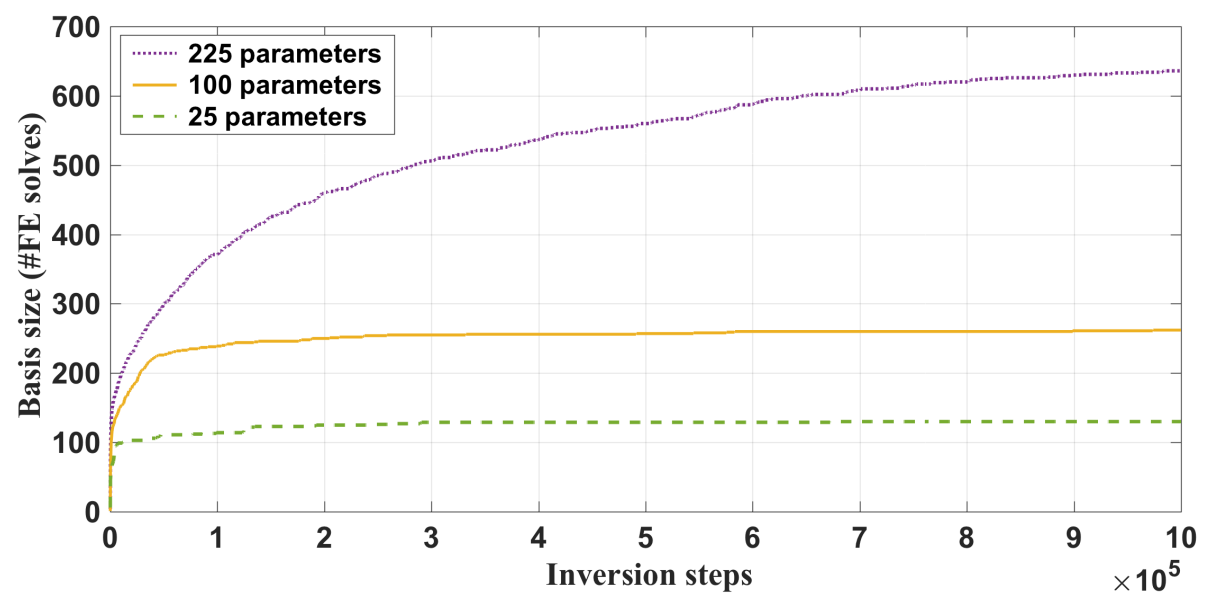

Figure 9. Number of FE problems solved during an inversion using RB strategy. Inversion for different number of parameters: 25 (solid line), 100 (dashed line) and 225 (dotted line).

\subsubsection{Other factors controlling basis size}

The smaller the basis, the smaller the computational cost involved in an inversion. Hence, the actual challenge is to create a basis which is small but still comprises all possible solutions we are interested in. The basis size is mainly determined by the region of the parameters space that is to be explored, therefore, all variables determining how the exploration takes place will influence the dimension of the basis. Besides the number of parameters (studied above), other variables that affect the basis size are: the error in the data (36) and the form of the proposal distribution (37).

If the error in the observables/data is large, the number of plausible models increases and so it does the parameter space for which accurate solutions are required from the surrogate. Consequently, the basis size will increase in order to include a larger number of possible solutions. Figure 10 shows the effect of different data error levels in (a) the random walk, (b) the posterior distribution and (c) the evolution of the basis size for three different $\sigma_{e}$ values: 1, 3 and 5\%. As expected, the range of variability of trial models increases with $\sigma_{e}$ (Fig. 10a) and the posterior PDF becomes wider (Fig. 10b), reflecting a poorer constraint on the parameter from the (bad quality) data.

The proposal distribution determines the way the parameter space is explored. If jumps between trial models are small (i.e. small $\sigma_{p}$ ), the parameter space is explored slowly (requiring long chains), but the acceptance rate is high (Tarantola, 2005; Gregory, 2005). Likewise, if the moves are large, the exploration of the parameter space is more efficient, but the acceptance rate decreases accordingly. The design of efficient proposals is thus crucial for the success of any MCMC algorithm and an important 

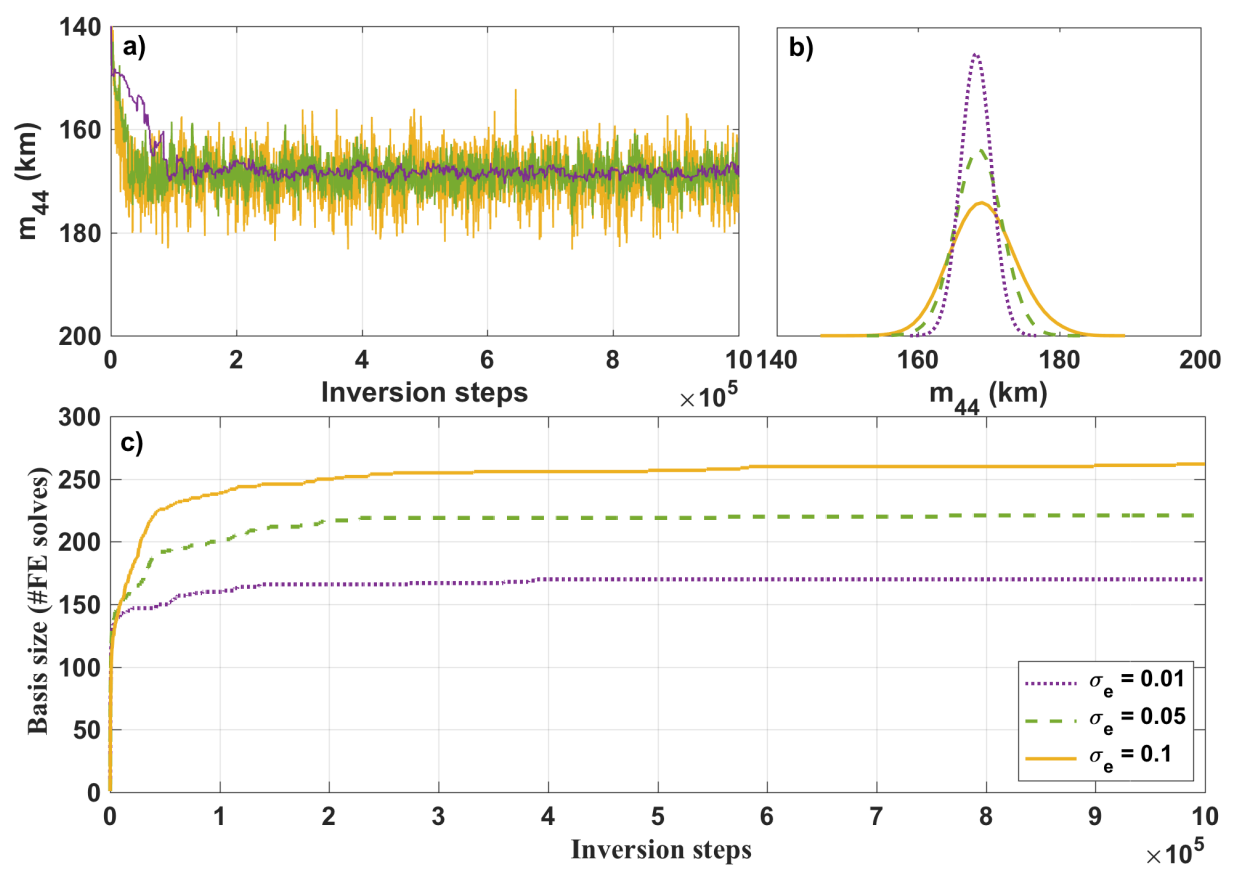

Figure 10. Implications of noise size in (a) random walk, (b) posterior distribution and (c) basis evolution size for three different noise levels: $\sigma_{e}=0.01$ (solid green), $\sigma_{e}=0.03$ (dashed red) and $\sigma_{e}=0.05$ (dotted grey).

research topic in the MCMC literature (Roberts et al., 1997; Roberts \& Rosenthal, 2001). Unsurprisingly, we also see a significant influence in terms of basis size. In Figure 11 the same simulation has been run for three different $\sigma_{p}$ values: (a) $5 \mathrm{~km}$ (b) $10 \mathrm{~km}$ and (c) $20 \mathrm{~km}$. The values of the samples that are accepted as part of the chain according to the Metropolis ratio $\Gamma$ are shown in grey, whereas all the samples proposed during the inversion are indicated in colors. Similar to the effect of increasing $\sigma_{e}$, larger values of $\sigma_{p}$ (i.e. large proposed moves) result in a larger domain for which accurate solutions are required from the surrogate, which in turn requires larger basis sizes. The size of the basis for each $\sigma_{p}$ case is also shown in Fig. 11 (bottom). The key point is that, even if a sample is not accepted, its forward problem has to be solved and, therefore, it may contribute to increase the basis size.

However, as in traditional MCMC approaches, there are alternatives to minimize the growth of the RB. Although beyond the scope of this paper, some options are adaptivity of the proposal (Haario et al., 1999, 2001), adaptivity of the RB tolerance (i.e. larger tolerance at the beginning and smaller tolerance when the chain starts converging) or a combination of both. Also, the use of multiple surrogates of different accuracy could in principle increase efficiency and reduce the overall size of the basis. For instance, a cheap surrogate could be used to evaluate whether or not the sample is accepted, and only those which are accepted (and then representative of the high probability region) will be recomputed with $\mathrm{FE}$ and used to enrich the basis (which keeps being improved for those models in the high probability regions). A similar approach has been used in Cui et al. (2014) and Lieberman et al. (2010). 

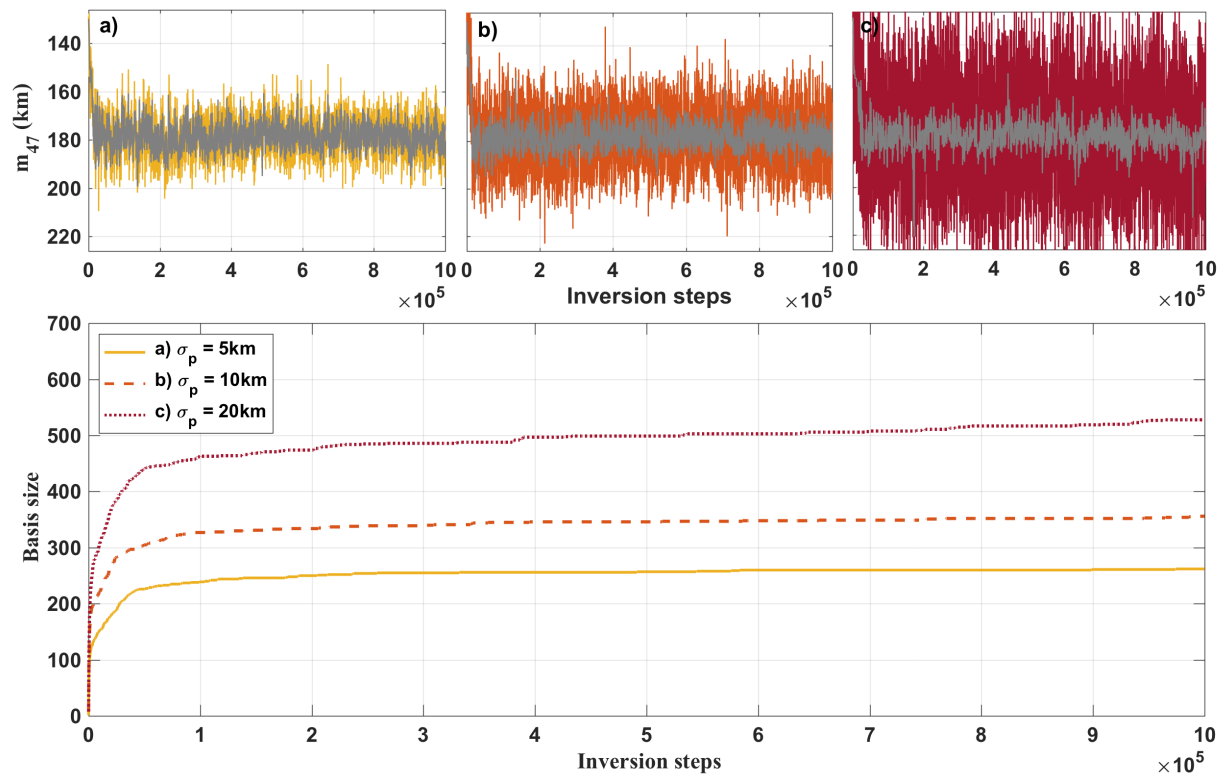

Figure 11. Implications of the proposal distribution in the posterior PDF (up) and in the basis evolution size (bottom) for three different proposals: (a) $\sigma_{p}=5 \mathrm{~km}$, (b) $\sigma_{p}=10 \mathrm{~km}$ and (c) $\sigma_{p}=20 \mathrm{~km}$. All proposed samples during the inversion (yellow, orange, red) and samples which are accepted (grey).

\subsection{Lithospheric structure beneath Africa}

In this section we apply our method to a larger, more realistic, example to determine the lithospheric structure and sublithospheric upper mantle flow pattern beneath the African continent and surroundings. While realistic in nature, we emphasize that this example is for illustrative purposes only.

The numerical domain is now spherical and discretized with 30x30x20 finite elements from the surface down to a depth of $1000 \mathrm{~km}$ depth. The vector of model parameters (LAB depths) contains 225 elements (i.e. the FE discretization is twice as fine as the LAB one). The input data are again the vertical velocities within the region of interest $\Gamma$ which for this example is larger, $\pm 100 \mathrm{~km}$ from the reference LAB value. The lithospheric structure (LAB depth and temperatures) of the reference model is based on the global lithospheric model by Afonso et al. (2019). Sublithospheric temperatures are computed based on the work of Stixrude and Lithgow-Bertelloni (2012) for a reference adiabatic gradient, with anomalies with respect to that gradient from the work of Steinberger and Becker (2018). Using this model as a reference, we compute the synthetic observables and contaminate them with Gaussian noise with a standard deviation of $15 \%$. Density and viscosity are computed as a function of temperature and pressure using equations (34) and (35), respectively.

We run the inversion algorithm for $10^{6}$ steps, from which the first $10^{4}$ samples were discarded as burn-in. The standard deviation of the proposal distribution was set at $\sigma_{p}=5 \mathrm{~km}$ and the threshold for the error estimator of the RB was set at $e_{\mathrm{RB}}=10^{-2}$. Mean values and standard deviations of the posterior PDF resulting from the inversion are shown in Figure 13. The corresponding numerical values as well as the plots of the random walks can be found in the Supplementary Material. 


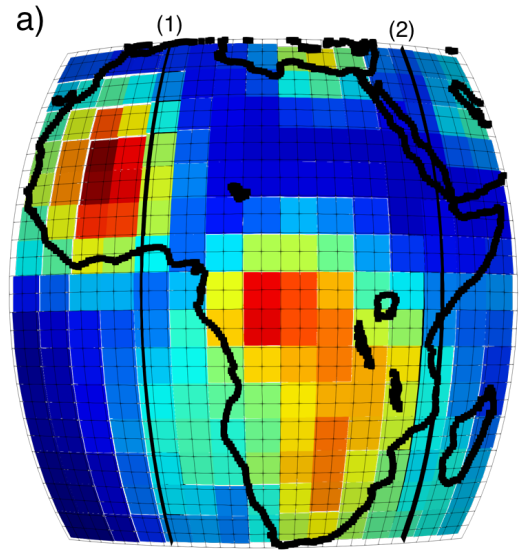

Reference LAB depth $(\mathrm{km})$ 446080100120140160180200220239 b)

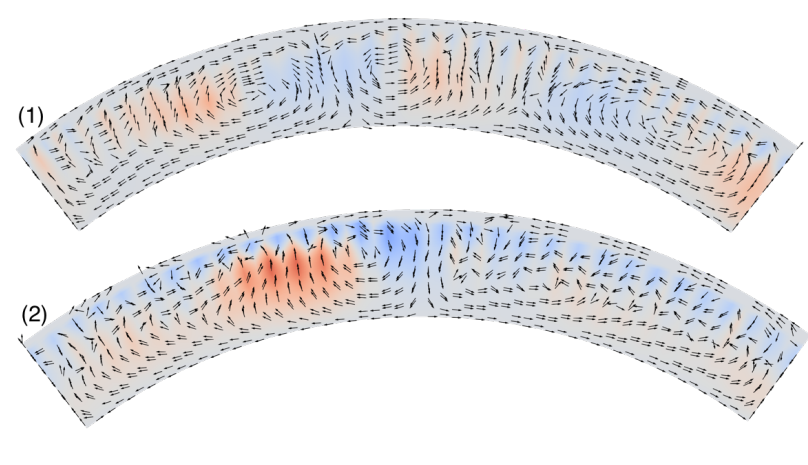

Modul normal velocity ( $\mathrm{cm} / \mathrm{year}$ )

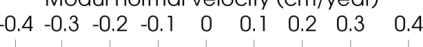

Figure 12. (a) Reference LAB discretized with 225 parameters. (b) Two vertical profiles showing the flow (velocity) structure.

a)

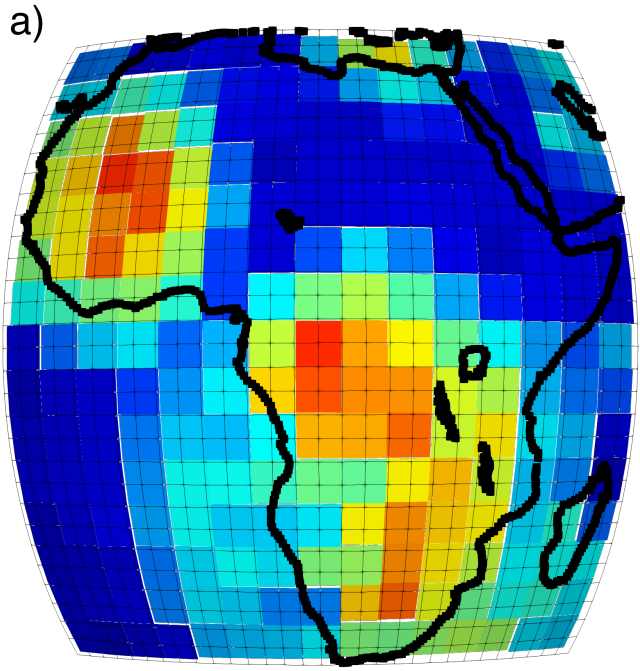

Mean of the recovered $L A B$ depth $(\mathrm{km})$

$4460 \quad 80 \quad 100120140160180200220239$ b)

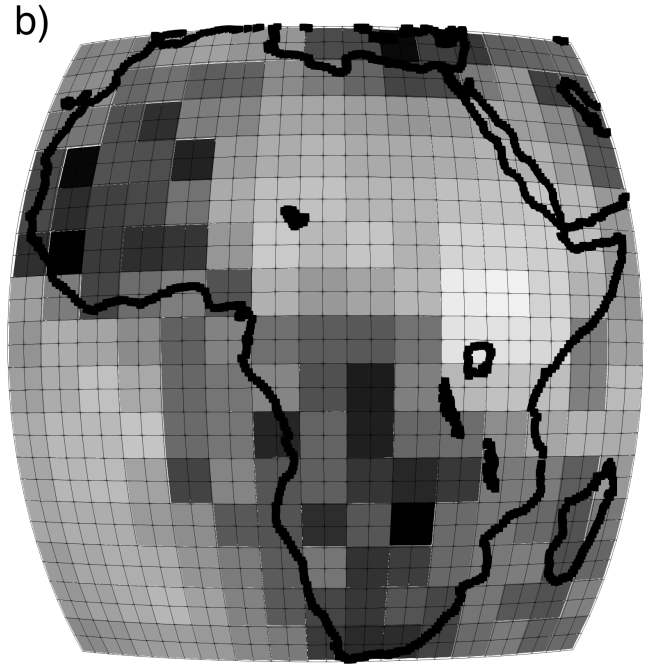

$\sigma$ of the recovered LAB depth $(\mathrm{km})$

46

Figure 13. (a) Mean and (b) standard deviation of the posterior PDF for each of the 225 parameters representing the LAB depth recovered during the inversion. 
The results in Fig. 13 and 12 demonstrate that the inversion succeeded in recovering the mean values of the true parameters. Wider posteriors are obtained in areas where the lithosphere is thicker, indicating that the observables are less sensitive to perturbations beneath these columns. This is not surprising, as these regions coincide with broad slow downwellings and small local perturbations of the LAB do not change the main velocity pattern or magnitude in any significant way. The narrowest distributions have standard deviations of around $5 \mathrm{~km}$ whereas the widest depict values of $46 \mathrm{~km}$. Detailed plots of each of the posteriors are shown in the Supplementary Material.

The efficiency of the method is again outstanding; to perform $10^{6}$ inversion steps, only $2360 \mathrm{FE}$ solves where required ( $0.24 \%$ of the total number of steps), or equivalently, one FE solve for every 400 inversions. It is worth emphasizing that in a real inversion context, more than $10^{6}$ would be typically run, which will increase even further the efficiency of the method.

\section{Some final remarks}

The efficiency gain provided by the implementation of a Reduced Basis approach into MCMC algorithms makes it practical to explore 3D full probabilistic inversions for the dynamic state of the Earth's interior. In particular, it opens up the possibility of combining multiple geodynamic and geophysical observables into a single inversion with global charaterization of uncertainties and account of non-linearities (e.g., Khan et al., 2008, 2011; Afonso, Fullea, Griffin, et al., 2013; Afonso, Fullea, Yang, et al., 2013; Afonso, Moorkamp, \& Fullea, 2016). It also facilitates the exploration of true "simulation-based inversions". By simulation-based we mean that complex forward problems (e.g. multi-phase thermomechanical simulations, full-waveform simulations), traditionally the realm of numerical modellers, can become the drivers of probabilistic inverse problems with no stringent requirements about simplifications of the physics (see e.g. Baumann et al., 2014; Baumann \& Kaus, 2015; Gebraad \& Fichtner, 2018). We consider this trend the future of inverse approaches in geoscience.

The proposed coupling of $\mathrm{MCMC}+\mathrm{RB}$ has one key advantage with respect to traditional methods in the construction of the reduced basis or surrogate: the basis is automatically "trained" on-the-fly to provide accurate solutions in the region (of the parameter space) that is most critical for the inversion scheme. This removes the need to perform "blind" offline experiments to construct a surrogate, which becomes harder and harder as the number of model parameters increases. The price to pay by merging the online and offline stages is that since the RB is built and updated during the inversion, the time to compute one particular inversion cannot be predicted in advance. It also introduces some subtle theoretical and practical difficulties. From a theoretical standpoint, since the surrogate effectively changes every time it is updated (i.e. the forward model changes), standard convergence analyses and proofs of ergodicity and detailed balance found in the literature (e.g. Yan \& Zhang, 2017) cannot be easily adopted. Given the numerical character of this work, here we do not attempt such analyses and/or proofs, but acknowledge that a general description and validation of the method will require an in-depth study of these properties; we reserve this for a future study.

From the practical point of view, the RB method provides a solution with a computational cost that depends on the size of the basis. Therefore, any efficient implementation of the method requires keeping the size of the basis small. In this paper we introduced two concepts that aid in keeping the size of the basis small: i) exploiting the convergent nature of MCMC algorithms and ii) the use of a goal-oriented error estimator to detect when the basis needs to be enriched. The first concept is intrinsic to the coupling of RB and MCMC and it can become very efficient in cases 
where information redundancy in the basis is monitored closely. For instance, certain solutions added to the basis during the initial stags of the inversion may become completely obsolete once the inversion has located the areas of high probability. This is because we are moving to a different region in the parameter space where previous solutions may be irrelevant. Additional tests (not shown here) demonstrated that even a simple SVD decomposition of the RB at specific intervals during the inversion is a very effective way of keeping the size of the basis small and removing redundant information in the basis. This become particularly important if the error estimator for updating the basis is not of the goal-oriented type.

The use of a tailored goal-oriented error (see Section 3.2.4) is a key ingredient of the present method and is applicable to a large number (but certainly not all) of inversion problems. For example, in the case of an inversion where dynamic topography needs to be computed, vertical strain rates at either the surface of the model or at the base of the mechanical lithosphere need to be predicted accurately (unless a free surface formulation is used). So the basis may be trained to describe properly that particular quantity and not the complete velocity field of the model. In other words, velocities outside the region of interest are allowed to be less accurate, only if their values do not influence the quantity of interest within the prescribed threshold error. This generally results in a basis significantly smaller than that which would be required to reproduce well the solution in the entire domain.

Finally, the current examples used a Newtonian formulation of the Stokes problem. However, if non-linear rheologies (e.g. diffusion and dislocation creep laws) are adopted, the RB approximation remains the same as that of the Newtonian case and no modification are required to the method presented here. The main difference is that the error estimation strategy should be set accordingly to account for the non-linear character of the problem (e.g., Huerta \& Díez, 2000).

\section{Conclusions}

We present an efficient implementation of a Reduced Basis (RB) solver for the solution of the Stokes flow within a probabilistic inversion framework. The proposed approach combines the efficiency of a RB-based surrogate solver for the Stokes problem with the generality of Markov Chain Monte Carlo methods for complex geophysicalgeodynamic inverse problems. In contrast to traditional implementations of RB, where the problem is split into two stages (i.e. offline and online), our approach takes advantage of the ergodicity and stationarity properties of MCMC formulations to create and update the surrogate on a single stage, during the inversion process.

The feasibility and efficiency of the combined RB+MCMC method are demonstrated using synthetic examples aimed at recovering the lithospheric structure in both illustrative and realistic scenarios. In all cases, the proposed method is able to successfully solve the probabilistic inverse problem using less than $1 \%$ of the computational cost needed in a standard MCMC approach. In other words, the combined $\mathrm{RB}+\mathrm{MCMC}$ method offers a staggering gain in computational cost of more than $99 \%$ compared to its standard MCMC counterpart. Moreover, these estimates are lower bounds and higher efficiency can be achieved in longer MCMC simulations due to the converging nature of the basis. The effect of a number of key parameters (e.g. dimension of the parameter space, data errors, tolerances used for the surrogate) on the performance of the method are also assessed.

The proposed method overcomes some important limitations of determinisitc geodynamic-geophysical inversions and makes possible the use simulation-based probabilistic inversion approaches for the physical and dynamic state of planetary interiors. Although our focus was on applications within the context of multi-observable prob- 
abilistic inversions (e.g., Afonso, Fullea, Yang, et al., 2013; Afonso, Rawlinson, et al., 2016), our method is general enough to find applications in problems such as uncertainty quantification/propagation, adjoint-based geodynamic inversions, sensitivity analyses in mantle convection problems as well as in other complex forward problems of geophysical interest (e.g. magnetotellurics, heat transfer, seismic tomography). We are currently working on some of them.

\section{Appendix A Extra FE solutions}

The criterion (9) used to accept or reject a proposed model (i.e. a new state in the chain) is based on comparing the value of the posterior for the proposed model, $\sigma\left(\mathbf{m}_{\text {new }}\right)$, with the posterior for the current state of the chain. The evaluation of the posterior requires the solution of a forward problem, which associated a specific error/accuracy. Since our RB approach builds and adapts the basis on the fly using error estimators, this accuracy may vary among successive forward problems. Consider the case when the error of the RB solution for a proposed model is above the predefined threshold and thus a high-fidelity FE solution is required. If the forward problem of the current state in the chain was accurately solved in a previous step with the RB surrogate, the comparison in (9) is now performed between a RB approximation (current) and a high-fidelity FE solution (new proposal). Since both of these solutions have different intrinsic accuracies/errors and misfits, the comparison is no longer objective and the Metropolis criterion is corrupted. In practice, significant differences in their respective errors is not uncommon and may result in a divergent chain and/or an inaccurate representation of the posterior. When this is the case, the random walk typically becomes "stuck" at some "good" model computed with the surrogate as new proposed models based on high-fidelity solutions tend to have higher misfits and therefore they are rejected. This difficulty does not exist in simpler approaches where a single surrogate is created offline and used unmodified during the inversion (e.g., Galbally et al., 2009; Wang \& Zabaras, 2005; Lieberman et al., 2010).

Now consider the opposite case, where the proposed model is accurately solved with the RB surrogate and the current model was solved with a high-fidelity FE solver. The comparison in (9) remains non-objective. However, unlike the previous case, the random walk generally does not get stuck here since the current model is the most accurate one (i.e. solved with the high-fidelity solver).

The problem described above can be avoided with a small modification to the original algorithm. Given the Markovian property of the chain (i.e. dependence only on the current state of the chain), the only strong requirement is that the comparison in (9) remains objective. Therefore the simplest solution requires that every time a proposed model is solved with FE, we also recompute (only if needed) the previous model with FE. In doing so, the comparison is always performed with solutions represented in the same space and exhibiting a similar accuracy. The recomputed FE solution of the previous model is only used for the comparison in (9) and is not added to the basis, as otherwise it would provide redundant information to it. While this simple approach guarantees that the comparison is objective, it has the drawback of requiring additional FE solutions. More efficient approaches can certainly be devised, but a complete description of them and their effect on the covergence and ergodicity of the chain are beyond the scope of this work and thus left for a forthcoming publication. 


\section{Acknowledgments}

We thank the positive and encouraging comments of Niklas Linde and two anonymous reviewers. OO, SZ and PD have been funded by the Spanish Ministry through grant DPI2017-85139-C2-2-R, by Catalan government through grant 2017-SGR-1278 and by the EU's Horizon 2020 research and innovation programme under the Marie Sklodowska-Curie grant agreement No 777778. JCA research has been funded by ARC Grant DP160103502. He also acknowledges support from the ARC Centre of Excellence Core to Crust Fluids Systems (CCFS), the Centre for Earth Evolution and Dynamics, Geoscience Australia and the European Space Agency via the "3D Earth - A Dynamic Living Planet". The data used in this work is available in http://doi.org/10.5281/zenodo.3615634.

\section{References}

Afonso, J. C., Fullea, J., Griffin, W. L., Yang, Y., Jones, A. G., Connolly, J. A. D., \& O'Reilly, S. Y. (2013). 3-D multiobservable probabilistic inversion for the compositional and thermal structure of the lithosphere and upper mantle. I: a priori petrological information and geophysical observables. Journal of Geophysical Research: Solid Earth, 118(5), 2586-2617. doi: 10.1002/jgrb.50124

Afonso, J. C., Fullea, J., Yang, Y., Connolly, J. A. D., \& Jones, A. G. (2013). 3D multi-observable probabilistic inversion for the compositional and thermal structure of the lithosphere and upper mantle. II: General methodology and resolution analysis. Journal of Geophysical Research: Solid Earth, 118(4), 1650-1676. doi: 10.1002/jgrb.50123

Afonso, J. C., Moorkamp, M., \& Fullea, J. (2016). Imaging the Lithosphere and Upper Mantle. In Integrated imaging of the earth: Theory and applications (pp. 191-218). doi: 10.1002/9781118929063.ch10

Afonso, J. C., Rawlinson, N., Yang, Y., Schutt, D. L., Jones, A. G., Fullea, J., \& Griffin, W. L. (2016). 3-D multiobservable probabilistic inversion for the compositional and thermal structure of the lithosphere and upper mantle: III. Thermochemical tomography in the Western-Central U.S. Journal of Geophysical Research: Solid Earth, 121(10), 7337-7370. doi: 10.1002/2016JB013049

Afonso, J. C., Salajegheh, F., Szwillus, W., Ebbing, J., \& Gaina, C. (2019). A global reference model of the lithosphere and upper mantle from joint inversion and analysis of multiple data sets. Geophysical Journal International, 217(3), 1602-1628. doi: 10.1093/gji/ggz094

Afonso, J. C., Zlotnik, S., \& Díez, P. (2015). An efficient and general approach for implementing thermodynamic phase equilibria information in geophysical and geodynamic studies. Geochemistry, Geophysics, Geosystems, 16(10), 3767-3777. doi: 10.1002/2015GC006031

Baumann, T. S., \& Kaus, B. J. (2015). Geodynamic inversion to constrain the nonlinear rheology of the lithosphere. Geophysical Journal International, 202(2), 1289-1316. doi: 10.1093/gji/ggv201

Baumann, T. S., Kaus, B. J., \& Popov, A. A. (2014). Constraining effective rheology through parallel joint geodynamic inversion. Tectonophysics, 631(C), 197-211. doi: $10.1016 /$ j.tecto.2014.04.037

Boschetti, F., \& Moresi, L. (2001). Interactive inversion in geosciences. GEOPHYSICS , 66 (4), 1226-1234. doi: 10.1190/1.1487069

Bui-Thanh, T., Willcox, K., \& Ghattas, O. (2008). Model Reduction for LargeScale Systems with High-Dimensional Parametric Input Space. SIAM Journal on Scientific Computing, 30(6), 3270-3288. doi: 10.1137/070694855

Bunge, H.-P., Hagelberg, C. R., \& Travis, B. J. (2003). Mantle circulation models with variational data assimilation: inferring past mantle flow and structure from plate motion histories and seismic tomography. Geophysical Journal International, 152(2), 280-301. doi: 10.1046/j.1365-246X.2003.01823.x 
Chinesta, F., \& Ladevèze, P. (2014). Separated Representations and PGD-Based Model Reduction (Vol. 554; F. Chinesta \& P. Ladevèze, Eds.). Vienna: Springer Vienna. doi: 10.1007/978-3-7091-1794-1

Colli, L., Ghelichkhan, S., Bunge, H.-P., \& Oeser, J. (2018). Retrodictions of mid paleogene mantle flow and dynamic topography in the atlantic region from compressible high resolution adjoint mantle convection models: Sensitivity to deep mantle viscosity and tomographic input model. Gondwana Research, 53, 252 - 272. doi: 10.1016/j.gr.2017.04.027

Cui, T., Marzouk, Y., \& Willcox, K. (2014). Data-driven model reduction for the Bayesian solution of inverse problems. International Journal for Numerical Methods in Engineering, 102(5), 966-990. doi: 10.1002/nme.4748

Florentin, E., \& Díez, P. (2012). Adaptive reduced basis strategy based on goal oriented error assessment for stochastic problems. Computer Methods in Applied Mechanics and Engineering, 225-228, 116-127. doi: 10.1016/j.cma.2012 .03 .016

Forte, A. M. (2007). Constraints on seismic models from other disciplines - implications for mantle dynamics and composition. In Treatise on geophysics (p. 805 858). Elsevier. doi: 10.1016/B978-044452748-6.00027-4

Forte, A. M., Woodward, R. L., \& Dziewonski, A. M. (1994). Joint inversions of seismic and geodynamic data for models of three-dimensional mantle heterogeneity. Journal of Geophysical Research: Solid Earth, 99(B11), 21857-21877. doi: 10.1029/94JB01467

Galbally, D., Fidkowski, K., Willcox, K., \& Ghattas, O. (2009). Non-linear model reduction for uncertainty quantification in large-scale inverse problems. International Journal for Numerical Methods in Engineering, 81(12), 1581-1608. doi: $10.1002 /$ nme. 2746

García-Blanco, R., Borzacchiello, D., Chinesta, F., \& Diez, P. (2017). Monitoring a PGD solver for parametric power flow problems with goal-oriented error assessment. International Journal for Numerical Methods in Engineering, 111(6), 529-552. doi: 10.1002/nme.5470

Gebraad, L., \& Fichtner, A. (2018). Bayesian Elastic Full-Waveform Inversion using Hamiltonian Monte Carlo. AGU Fall Meeting(Abstract S31D-0525).

Gilks, W. R., Richardson, S., \& Spiegelhalter, D. J. (1996). Markov Chain Monte Carlo in Practice.

Gregory, P. (2005). Bayesian Logical Data Analysis for the Physical Sciences.

Haario, H., Saksman, E., \& Tamminen, J. (1999). Adaptive proposal distribution for random walk Metropolis algorithm. Computational Statistics, 14(3), 375. doi: $10.1007 / \mathrm{s} 001800050022$

Haario, H., Saksman, E., \& Tamminen, J. (2001). An Adaptive Metropolis Algorithm. Bernoulli, 7(2), 223-242. doi: 10.2307/3318737

Hastings, W. K. (1970). Monte Carlo sampling methods using Markov chains and their Applications. Biometrika, 57(1), 97-109.

Hesthaven, J. S., Rozza, G., \& Stamm, B. (2016). Certified Reduced Basis Methods for Parametrized Partial Differential Equations. Cham: Springer International Publishing. doi: 10.1007/978-3-319-22470-1

Huerta, A., \& Díez, P. (2000). Error Estimation Including Pollution Assessment for Nonlinear Finite Element Analysis. Computer Methods in Applied Mechanics and Engineering, 181(1-3), 21-41. doi: 10.1016/S0045-7825(99)00071-7

Hughes, T. J. R. (1987). The Finite Element Method: Linear Static and Dynamic Finite Element Analysis. Prentice-Hall.

Ito, K., \& Ravindran, S. (1998). A Reduced-Order Method for Simulation and Control of Fluid Flows. Journal of Computational Physics, 143(2), 403-425. doi: 10.1006/jcph.1998.5943

Kaipio, J. P., \& Somersalo, E. (2005). Statistical and Computational Inverse Problems (Vol. 160). New York: Springer-Verlag. doi: 10.1007/b138659 
Kaipio, J. P., \& Somersalo, E. (2007). Statistical inverse problems: Discretization, model reduction and inverse crimes. Journal of Computational and Applied Mathematics, 198(2), 493-504. doi: 10.1016/j.cam.2005.09.027

Khan, A., Connolly, J. A. D., \& Taylor, S. R. (2008). Inversion of seismic and geodetic data for the major element chemistry and temperature of the Earth's mantle. Journal of Geophysical Research, 113(B9), B09308. doi: 10.1029/2007JB005239

Khan, A., Zunino, A., \& Deschamps, F. (2011). The thermo-chemical and physical structure beneath the North American continent from Bayesian inversion of surface-wave phase velocities. Journal of Geophysical Research, 116(B9), B09304. doi: 10.1029/2011JB008380

Li, D., Gurnis, M., \& Stadler, G. (2017). Towards adjoint-based inversion of timedependent mantle convection with non-linear viscosity. Geophysical Journal International, 209(1), 86-105. doi: 10.1093/gji/ggw493

Lieberman, C., Willcox, K., \& Ghattas, O. (2010). Parameter and State Model Reduction for Large-Scale Statistical Inverse Problems. SIAM Journal on Scientific Computing, 32(5), 2523-2542. doi: 10.1137/090775622

Linde, N., Ginsbourger, D., Irving, J., Nobile, F., \& Doucet, A. (2017). On uncertainty quantification in hydrogeology and hydrogeophysics. Advances in Water Resources, 110(October), 166-181. doi: 10.1016/j.advwatres.2017.10.014

Malinverno, A., \& Briggs, V. A. (2004). Expanded uncertainty quantification in inverse problems: Hierarchical Bayes and empirical Bayes. Geophysics, 69(4), 1005-1016. doi: 10.1190/1.1778243

Metropolis, N., Rosenbluth, A. W., Rosenbluth, M. N., Teller, A. H., \& Teller, E. (1953). Equation of State Calculations by Fast Computing Machines. The Journal of Chemical Physics, 21(6), 1087-1092. doi: 10.1063/1.1699114

Mosegaard, K., \& Tarantola, A. (2002). Probabilistic Approach to Inverse Problems. In International handbook of earthquake and engineering seismology (Part A) (pp. 237-265).

Peherstorfer, B., Willcox, K., \& Gunzburger, M. (2018). S Survey of Multifidelity Methods in Uncertainty Propagation, Inference, and Optimization. SIAM Review, 60(3), 550-591. doi: 10.1137/16M1082469

Prud'homme, C., Rovas, D., Veroy, K., Machiels, L., Maday, Y., Patera, A. T., \& Turinici, G. (2002). Reliable Real-Time Solution of Parametrized Partial Differential Equations: Reduced-Basis Output Bound Methods. Journal of Fluids Engineering, 124(1), 70. doi: 10.1115/1.1448332

Quarteroni, A., Manzoni, A., \& Negri, F. (2016). Reduced Basis Methods for Partial Differential Equations (Vol. 92). Cham: Springer International Publishing. doi: 10.1007/978-3-319-15431-2

Ravindran, S. (2000). A reduced-order approach for optimal control of fluids using proper orthogonal decomposition. International Journal for Numerical Methods in Fluids, 34(5), 425-448.

Roberts, G. O., Gelman, A., \& Gilks, W. R. (1997). Weak convergence and optimal scaling of random walk metropolis algorithms. The Annals of Applied Probability, 7(1), 110-120.

Roberts, G. O., \& Rosenthal, J. S. (2001). Optimal scaling for various MetropolisHastings algorithms. Statistical Science, 16(4), 351-367. doi: 10.1214/ss/ 1015346320

Rozza, G., Huynh, D. B. P., \& Manzoni, A. (2013). Reduced basis approximation and a posteriori error estimation for Stokes flows in parametrized geometries: roles of the inf-sup stability constants. Numerische Mathematik, 125(1), 115-152. doi: 10.1007/s00211-013-0534-8

Rozza, G., Huynh, D. B. P., \& Patera, A. T. (2007). Reduced basis approximation and a posteriori error estimation for affinely parametrized elliptic coercive partial differential equations. Archives of Computational Methods in Engineering, 

15(3), 1-47. doi: 10.1007/BF03024948

Schubert, G., Turcotte, D. L., \& Olson, P. (2004). Mantle Convection in the Earth and Planets. Cambridge University Press.

Serafin, K., Magnain, B., Florentin, E., Parés, N., \& Díez, P. ～(2017). Enhanced goal-oriented error assessment and computational strategies in adaptive reduced basis solver for stochastic problems. International Journal for Numerical Methods in Engineering, 110(5), 440-466. doi: 10.1002/nme.5363

Simmons, N. A., Forte, A. M., Boschi, L., \& Grand, S. P. (2010). GyPSuM: A joint tomographic model of mantle density and seismic wave speeds. Journal of Geophysical Research, 115(B12), B12310. doi: 10.1029/2010JB007631

Simmons, N. A., Forte, A. M., \& Grand, S. P. (2009). Joint seismic, geodynamic and mineral physical constraints on three-dimensional mantle heterogeneity: Implications for the relative importance of thermal versus compositional heterogeneity. Geophysical Journal International, 177(3), 1284-1304. doi: 10.1111/j.1365-246X.2009.04133.x

Steinberger, B., \& Becker, T. W. (2018). A comparison of lithospheric thickness models. Tectonophysics, 746, 325-338. doi: 10.1016/j.tecto.2016.08.001

Stixrude, L., \& Lithgow-Bertelloni, C. (2012). Geophysics of Chemical Heterogeneity in the Mantle. Annual Review of Earth and Planetary Sciences, 40(1), 569-595. doi: 10.1146/annurev.earth.36.031207.124244

Tarantola, A. (2005). Inverse Problem Theory and Methods for Model Parameter Estimation. SIAM.

Titus, W. J., Titus, S. J., \& Davis, J. R. (2017). A Bayesian approach to modeling 2D gravity data using polygons. Geophysics, 82(1), G1-G21. doi: 10.1190/ GEO2016-0153.1

Tork Qashqai, M., Afonso, J. C., \& Yang, Y. (2018). Physical State and Structure of the Crust Beneath the Western-Central United States From Multiobservable Probabilistic Inversion. Tectonics, 37(9), 3117-3147. doi: 10.1029/2017TC004914

Tork Qashqai, M., Carlos Afonso, J., \& Yang, Y. (2016). The crustal structure of the Arizona Transition Zone and southern Colorado Plateau from multiobservable probabilistic inversion. Geochemistry, Geophysics, Geosystems, 17(11), 4308-4332. doi: 10.1002/2016GC006463

van Dinther, Y., Gerya, T. V., Dalguer, L. A., Mai, P. M., Morra, G., \& Giardini, D. (2013). The seismic cycle at subduction thrusts: Insights from seismothermo-mechanical models. Journal of Geophysical Research: Solid Earth, 118(12), 6183-6202. doi: 10.1002/2013JB010380

Wang, J., \& Zabaras, N. (2005). Using Bayesian statistics in the estimation of heat source in radiation. International Journal of Heat and Mass Transfer, 48(1), 15-29. doi: 10.1016/j.ijheatmasstransfer.2004.08.009

Yan, L., \& Zhang, Y.-X. (2017). Convergence analysis of surrogate-based methods for bayesian inverse problems. Inverse Problems, 33(12), 125001. doi: 10.1088/ $1361-6420 /$ aa9417

Zienkiewicz, O. C., Taylor, R. L., \& Zhu, J. Z. (2005). The Finite Element Method: its Basis and Fundamentals. Elsevier. doi: 10.1016/C2009-0-24909-9 